\title{
15. PHYSICAL STUDY OF COMETS, MINOR PLANETS AND METEORITES (L'ÉTUDE PHYSIQUE DES COMĖTES, PETITES PLANÈTES ET DES MÉTÉORITES)
}

PRESIDENT: A. H. Delsemme.

VICE-PRESIDENT: N. B. Richter.

SECRETARY: J. Rahe.

ORGANIZING COMMITTEE: E. Anders, C. Arpigny, L. Biermann, O. V. Dobrovolskij, B. D. Donn, T. Gehrels, E. Roemer, V. Vanysek, F. Whipple.

\section{INTRODUCTION}

(a) The enlargement of the scope of Commission 15 that was decided in 1973, partially stems from the recognition that comets, minor planets and meteorites give closely interrelated clues on the origin of the solar system. The related increase of interest as well as the success of several novel techniques of observations, probably explains the considerable expansion of the field during the last three years.

(b) Sections 1, 2, 3 of this Report were written by the President from draft contributions provided by Brandt, Chapman, Dobrovolskij, Gehrels, Greenberg, Haser, Herzberg, Huebner, Jackson, Keller, Konopleva, Levin, R. Lüst, Marsden, Mendis, Morrison, O'Dell, Rahe, Sekanina, Shul'man, Wallis and Zellner. He wishes to express his gratitude for their help. To keep continuity and emphasize fundamental problems, subject matters have been divided into headings set by the physical phenomena and not by the observational techniques. In particular, since this report is expected to be a critical review and not a source book for bibliography, the traditional section on observations quoted without interpretations, has been omitted; most of these observations can be found in Astronomy and Astrophysics Abstracts, under 098 (Minor Planets) or 103 (Comets: listed objects). The concise reference system used in Sections 1 through 3 is described at the end.

(c) Section 4 on meteorites was written by L. Grossman; he has used a slightly different reference system described in his introduction. His important contribution is acknowledged gratefully.

(d) Proceedings of International or Regional Colloquia are an important source of information on current research. The following meetings have been devoted to or connected with the physical study of comets and minor planets: IAU Colloquia C22, C25, C31, C39; Kiev Colloquium C8; Liège Colloquium C20; NASA Workshops C1 and C2. Unfortunately, most of these proceedings are not yet available at the time of this writing. Those of $C 22$ were published in $1974, C 2$ in $1975, C 25$ early in 1976.

(e) Two new observatories are mostly devoted to cometary research. One was dedicated on August 19, 1974 at Socorro, New Mexico, U.S.A., operated jointly by NASA (J. Brandt) and the Institute of Mining and Technology in Socorro, New Mexico (S. A. Colgate); the Kiev University Observatory for Cometary and Radioastronomical Research was dedicated on 4 October 1974, at Lesniki near Kiev (S. K. Vsekhsvjatsky).

(f) Comet Kohoutek (1973 XII) became the most observed comet and brought out the most important advances. Icarus (23, No. 4) had an entire issue (22 papers) devoted to 1973 XII.

(g) Reviews are sharply separated by the passage of 1973 XII. Before, Delsemme (10.102.011) reviews the production of gas and dust in comets; Marsden (12.102.008) reviews orbital and statistical data, connecting them with the physical study of comets, and Vanysek (12.102.013) the composition of cometary dust. After, Delsemme (Icarus 24, 34) makes the first extensive comparison between comets, carbonaceous chondrites, meteor spectra, meteor shower ions and lunar soil; Mendis and Ip ( $A S S$, in press) review the cometary atmospheres; 
Jackson ( $J$. Photochem., in press) reviews cometary photochemistry. During $C 25$, sixteen different cometary topics were also reviewed by Arpigny, Brandt, Delsemme, Everhart, Herbig, Herzberg, Jackson, Keller, Ney, Marsden, Rasool, Roemer, Schmidt, Sekanina, Snyder, Vanysek.

(h) The Atlas Cometas-Viento Solar published in 1973 by the Observatorio Astronomico of the University of Cordoba (Argentina) gives isophotometry curves for 13 comet photographs, from $1963 \mathrm{~V}$ to 1969 IX. Richter and Högner announce the publication of part II of their Isophotometric Atlas of Comets (in press); 88 isophote diagrams are given from 120 plates of all bright comets from 1956 to 1974. No progress report on Part II of the Atlas of Cometary Spectra has been received so far from Liège.

(i) Marsden's annual Comet Report in QJRAS, supersedes Roemer's Comet Notes, discontinued in Mercury. $K M D$ is still published in Dushambe (Instit. Astrophys.) and $K T K$ by Kiev University. Konopleva, Nazarchuk and Shul'man have in press (Naukova Dumka, Kiev 1976) a monograph on The Surface Brightness of Comets. Vsekhsvjatsky and Ilchishina published The Physical Characteristics of the 1965-1970 Comets (Nauka, Moscow, 1974).

(j) Asteroid 433 Eros was the subject of a special campaign near the time of its very close passage to Earth in early 1975 . Icarus (28, No. 1) is publishing an entire issue (15 papers) devoted to this object. A series of 5 papers devoted to an earlier campaign to study 1685 Toro was published in 1973 in the $A J(78$, No. 6$)$.

(k) A review of physical data on minor planets current to the beginning of 1975 was published by Chapman, Morrison, and Zellner (Icarus 25, 104), and a data bank on physical observations is being prepared by Zellner and others at the University of Arizona. The problem of asteroids as parent bodies of the meteorites was reviewed by Chapman (Geochim. Cosmochim. $A c t a$, in press). Reviews on meteorites are noted in the introduction of Section 4 and listed in a special reference list.

\section{COMETS}

\section{A. Structure and Nature of the Cometary Nucleus}

(a) Issues about the structure of the cometary nucleus have been rather clarified. Although Lyttleton $(A S S 34,491)$ continues to argue that comets have no solid nucleus, he has never properly refuted any of the three major former criticisms against his loose 'sandbank' model, based on total mass loss, collapse, and gas drag from observed production rates (Whipple, $C 20, C 25)$, whereas all his criticisms of the icy conglomerate model seem to have been satisfactorily answered (see $I A U$ Report 1973 , p. 182 and Delsemme, C22). In particular, neither the existence of the non-gravitational forces nor the interpretation of their nature is much in doubt any more (Marsden 12.102.008); therefore, since the reactive force of the vaporizing gas is transmitted to the center of mass of the whole nucleus without blowing it away, its cohesive strength cannot be nil, which rules out the loose type of 'sandbank' model. The dense type, held together by gravitation, would have collapsed, if not blown away earlier by vaporization gases (O'Dell 09.102.014).

(b) However, the cohesive strength of the nucleus must admittedly be very weak, as evidenced by sungrazing comets split by tidal disruption; this is also suggested by brightness outbursts and by the brittleness and low density of those bright meteors that presumably are cometary debris (Whipple, C20).

(c) The chemical nature of the nucleus is still rather uncertain, although recent results show some progress. The basic difficulty is that all direct observational data come from those by-products of the nuclear disintegration that have already reached coma and tail, and the still uncertain history of this disintegration must be first understood and reconstructed. However, the non-volatile fraction is consistent with silicate dust (Section E) which is also suggested by comparison with meteors and meteorites as well as by solar elementary abundances (Delsemme, Icarus 24, 95), although Vanysek et al. (ASS 33, L19) speculate on the possibility of formaldehyde polymers as a major constituent of the dust. The puzzling observation of an essentially 
iron-free (therefore, strongly differentiated) non-volatile material, entering the Earth's atmosphere on a comet-type orbit (Harvey, $A J 79,333$ ) must also be kept in mind, in contrast to the solar abundances suggested by the stream meteors associated with known cometary orbits (see Commission 22 Report).

It seems however more and more likely that water (ice or snow) is one of the major constituents of the volatile fraction: Delsemme and Rud(10.102.008) give eight arguments; four of these imply that water controls the vaporization rate of several short-period comets (this does not exclude solid hydrates of gases, whose vaporization is controlled by their water-ice lattice). Previously, Marsden had empirically studied the dependence on heliocentric distances of the non-gravitational forces (NGF) affecting the motion of P/Schwassmann-Wachmann 2. Delsemme $(C 22$, p. 313) demonstrates that the best fit to this dependence law is given by a vaporization whose temperature is controlled by water ice (or solid hydrates), because it produces a sharp drop in the sublimation rates at the right distance. Next, Marsden et al. ( $A J$ $78,211)$ using a NGF dependence on distance that models vaporization, find the sudden drop in NGF consistently somewhere in-between 1 and $4 \mathrm{AU}$ for many short-period orbits; the best fit for the most significant comets is between 1.5 and $2.8 \mathrm{AU}$. Theory predicts $2.8 \mathrm{AU}$ for a water-ice nucleus of equal albedo in the visible $A_{\mathrm{v}}$ and in infrared $A_{i r}$, and less than $2.8 \mathrm{AU}$ if $A_{\mathrm{V}}>A_{\text {ir }}$ (inequality that has been predicted from brightness laws, Delsemme, $A L 14,163$ ); whereas anything slightly more volatile than water pushes far away the sudden drop in NGF: $8 \mathrm{AU}$ for $\mathrm{NH}_{3}, 11 \mathrm{AU}$ for $\mathrm{CO}_{2}$. Marsden and Sekanina $(A J 79,413)$ also show that the transverse NGF on $\mathrm{P} /$ Encke has diminished since its maximum around 1820 , possibly because of physical deactivation combined with axis precession. This deactivation of P/Encke is confirmed by Delsemme and Rud (10.102.008). Assuming that the production rates of 1969 IX and $1970 \mathrm{II}$ is controlled by water vaporization and using observations of the nuclear brightness at large distances, they derive nuclear Bond albedos of 0.6 to 0.7 in the visible, and nuclear radii of 2 to $4 \mathrm{~km}$, whereas they do not find an acceptable albedo and radius for $P / E n c k e$, without introducing a supplementary assumption, namely, either that its vaporization is controlled by a stuff much less volatile than water, or that no more than $10 \%$ of its surface area is covered by water snow.

For short-period comets, a consistent picture requiring no major constituent more volatile than water seems therefore to have emerged, whereas for long-period comets, a more volatile stuff is not unlikely. In particular, the statistical differences in the brightness laws in total light, mentioned by Oort and Schmidt (1951) between 'new,' young, older, and very old comets, are likely to come from the different distances at which these four groups have a sharp vaporization drop (Delsemme, C20). Of course the radiative steady state used 25 years ago (Levin's law) is no more acceptable; as a matter of fact, the drop occurs at that distance where radiation and vaporization exchange their roles (Delsemme, C22). Therefore the observational data collected for 60 comets by Bobrovnikoff and later by Schmidt should be reconsidered to establish the statistical distance of the vaporization drop for those four cometary groups defined by their orbital energy. In the absence of such an endeavor, Delsemme $(C 2)$ mentions that the light curve of 1973 XII before perihelion suggests a vaporization drop displaced beyond $4 \mathrm{AU}$, that is an icy conglomerate slightly more volatile than water. However, the difficulties of using light curves are serious (Section F).

The breakthrough in radioastronomical observations, reported in Section C, has yielded for the first time molecules that are undisputably stable enough to survive for aeons in the icy conglomerate: $\mathrm{H}_{2} \mathrm{O}, \mathrm{HCN}$ and $\mathrm{CH}_{3} \mathrm{CN}$; the abundance of the latter two molecules in the snows is unknown but they do not seem major constituents in $1973 \mathrm{XII}$; using these results as well as the rocket observations of ultraviolet resonance lines of the elements $\mathrm{O}, \mathrm{C}$ and $\mathrm{H}$, Delsemme $(C 20, C 25)$ and Shimizu (C25) speculate on the probable chemistry of the snows.

\section{B. Origin and Final Outcome of Comets}

\section{Existence of Oort's Cloud}

Marsden and Sekanina $(10.102 .038)$ note that the best available original orbits of quasiparabolic comets concentrate their aphelia near $50000 \mathrm{AU}$, reducing the distance but totally 
confirming the existence of Oort's sharp peak in $a^{-1}$ ( $a$ : semi-major axis) in the distribution of the orbits, that is, the existence of 'new' comets coming straight from the Öpik-Oort's big reservoir. Therefore, all physical theories on the origin of comets should now first explain the sharp peak in $a^{-1}$; so far, Oort only seems to have provided a semi-quantitative explanation. However, Lyttleton (12.102.016) neglecting LePoole and Katget's careful reply to his first criticisms (reply already mentioned, p. 144 in IAU report 1970), criticizes again the same inferences never made by Oort. In particular, Oort never suggested the existence of a 'shell' of comets whose maximum density would be detected by the peak in $a^{-1}$; nor that all comets in this peak are 'new.' On the other hand, Lyttleton does not offer any alternate explanation of the surprising concentration of the binding energies within such a small $\Delta a^{-1}$, although he is clearly aware that these binding energies would scatter over a range more than ten times as large, had most of the comets gone (even only once) through the inner solar system before.

\section{Origin of Oort's Cloud}

The present existence of Oort's cloud does not imply that we understand its origin or its age. The primeval belt of comets hypothesized by Oort, was pushed first (Kuiper, Whipple) from the asteroid belt to the range of Uranus-Neptune. Opik (09.107.017) explores extensively a model describing the formation, first of comets, then of planets, in pre-planetary rings of the residual solar nebula, with subsequent ejection, chiefly by Jupiter, of the comets to Oort's cloud. Vsekhsvjatsky's hypothesis (still being developed, $P K F 10$ ) that all comets were formed by volcanic eruptions on the planets or satellites, is not in formal contradiction with a recent origin $\left(<10^{8} \mathrm{yr}\right)$ of those comets coming (back?) from $50000 \mathrm{AU}$. However, the low efficiency of filling up Oort's cloud (whether by ancient planetary perturbations or recent eruptions) suggests to explore other possibilities. Cameron (09.107.407) proposes that Oort's cloud could have been created at its present distance, by nebular satellites or rings of the primeval nebula. Reeves (11.102.015) thinks that comets might result from the continuous condensation of the solar wind at large distances from the sun.McCrea (Nature 255, No. 5505, p. 96) suggests that comets were formed in Oort's cloud, but as recently as 1 or 2 million years ago, when the solar system passed through the compression lane which lies inside the Orion arm of the Galaxy. Urey (09.102.004) also suggests repeated periods during which cometary collisions with the earth were more probable: were tektites produced by cometary impacts? Greenberg $(A p J 189$, L81) believes that the balance explaining the depletion of $\mathrm{O}, \mathrm{C}, \mathrm{N}$ from the interstellar medium cannot be found in fine dust, but in 'snowballs' that could be either smaller than or as large as cometary nuclei. Tinsley $(A J 192,629)$, Tinsley and Cameron $(A S S 31,31)$ think that the total comet population of the Galaxy could be large enough to hide a significant fraction of metals and $\mathrm{C}, \mathrm{N}, \mathrm{O}$. Whipple $(A J \mathbf{8 0}, 525)$ cannot rule out this possibility, from present cometary statistics. The striking correlation between molecules observed in comets and in interstellar space is often mentioned but remains to be explained. O'Dell (09.102.014) shows that many independent grains would collapse to a single assemblage if it is to survive into the inner solar system. In the condensing process of the solar nebula, interstellar grains might not have been destroyed, but after an unknown thermal history, could have accreted into comets in the outer rings. Delsemme $(C 2 O)$ suggests that the ratios $\mathrm{C} / \mathrm{O}$ and $\mathrm{H} / \mathrm{O}$ of the production rates deduced from the resonance lines could be used as cosmothermometer. If present values are meaningful, they suggest an accretion temperature of $110^{\circ} \pm 60^{\circ} \mathrm{K}$ for the cometary nuclei. Whipple $(C 20)$ speculates that, if the primitive atmosphere of the terrestrial planets was eliminated by the T-Tauri phase of the Sun, the life-giving elements (C, N, O) of the Earth may well have been accumulated at a later stage by direct hits from comets and asteroids crossing the inner solar system, while being dispersed by planetary perturbations. Guseinov and Vanysek $(A S S 28, \mathrm{~L} 11)$ and Whipple $(A J 80,525)$ doubt the importance of the cometary influx on neutron stars as a source of gamma ray bursts, proposed by Harvit and Salpeter (10.102.020).

\section{The Origin of Short-Period Comets}

The origin of Short-Period Comets remains in dispute. Vsekhsvjatsky (PKF 10) still develops far-reaching consequences of his eruptive theory, which reverses the orbital evolution from long-period to short-period comets. However, Everhart's (10.102.012) numerical study on the 
evolution of $10^{6}$ orbits, reproduces remarkably well the distribution of periods of short-period comets, from hundreds of interactions with Jupiter resulting in capture of formerly parabolic orbits. The fact that those captures are from direct orbits with perihelia near Jupiter, answers Vsekhsvjatsky's major arguments against capture. Joss (09.102.013) claims with Vsekhsvjatsky that short-period comets are much too numerous to be explained by capture, but Delsemme (10.102.022) answers that their number is consistent with the capture rate of those intermediate-period comets with perihelion near Jupiter, whose large number can be predicted by orbital diffusion from Oort's cloud. These comets are much more numerous than those observable from the Earth (perihelion $q<2 \mathrm{AU}$ ), because they accumulate without vaporization for $q>4$ AU. Discussing several ideas of comet origins, Everhart (09.102.012) shows that some of the comets that would form in the Jupiter-Saturn region can be stabilized for some time in Trojan, horseshoe and mid-range orbits; eventually, they can decay into short-period orbits, but can never become long-period comets and cannot explain Oort's sharp peak in $a^{-1}$. Arguments for a cometary cosmogony in Laplace's style are also put forward by Radzievsky and Tomanov $(A V 7$, No. 2,73 and $83 ; 8$, No. 2,$87 ; 9$, No. 1$)$.

\section{Final Outcome}

A large fraction of the comets that go through the solar system are of course eventually ejected on hyperbolic orbits (Everhart 10.102 .012 ) whereas a very small fraction disappears by direct hits on the planets. Those that survive for a while, with a perihelion $\leq 5 \mathrm{AU}$, decay fast and provide a large (possibly the only serious) contribution to the interplanetary gas and dust (See Report of Commission 22). However, the final outcome is not clear. When comets become inactive, do they totally disappear into dust, or do they leave a compact core of non-volatile material that might survive (on unstable orbits) for a few million years? Shul'man (08.102.027) argues that the surface can quickly become covered by an insulating layer of dust, composed of those particles too large to be lifted from the nucleus. O'Dell $(C 25)$ uses observed low gas-todust ratios to argue that this insulating crust is usually thick. Some breaking down may take place but one or several chunks of solid material may be left over; alternately, a primeval core could also exist. A test is given by the final behavior of the NGF (Sekanina, C12, p. 423); if they fade away, they indicate a non-volatile core; if the NG acceleration grows when the comet's brightness fades away, it indicates total decay into dust. So far, Marsden et al.'s results show a trend of decreasing NGF, suggesting the existence of a non-volatile core for several comets; P/Encke seems one of the best examples. Inactive cometary nuclei must be left on rather unstable orbits (because of their eccentricity) but some of those that have decayed during the last million years should still be around, and the largest could be detected as asteroids. Whipple (10.098.024) discusses whether the fifteen Apollo asteroids could be inactive comet nuclei.

\section{The Neutral Coma}

For the first time, radioastronomical observations of comets have yielded results. Three stable parent molecules of observed radicals have been identified.

\section{The mm Range}

In the $\mathrm{mm}$ range, $\mathrm{CH}_{3} \mathrm{CN}$ was identified in Comet Kohoutek $1973 \mathrm{XII}$ by Ulich and Conklin (Nature 248,121 ) in the $J=6-5$ transitions of the vibrationally excited state $v_{8}=1$. Later, Buhl et al. (C25) probably identified very weak $J=5-4$ transitions in the same excited state, as well as in the vibrational ground state; this comes as a warning that a Boltzmann distribution based on the observations by Ulich and Conklin would yield-a serious overestimate of the $\mathrm{CH}_{3} \mathrm{CN}$ production rate. Huebner et al. (Icarus 23,580) identified $\mathrm{HCN}$ in the $J=1-0$ transitions in 1973 XII. Jackson et al. (C25) probably found the ground state $J=6-5$ rotationvibration transition of $\mathrm{H}_{2} \mathrm{O}$ in $1974 \mathrm{~b}$, but not in 1973 XII. If substantiated, this is the first direct observation in the coma, of a major. component of the nucleus. At small heliocentric distances, Huebner et al. also detect multiple Doppler shifts in the spectrum of HCN, interpreted as jets with velocities up to $\sim 3 \mathrm{~km} \mathrm{~s}^{-1}$. 


\section{The cm Range}

In the $\mathrm{cm}$ range, radicals $\mathrm{OH}$ and $\mathrm{CH}$, earlier observed only in the optical spectra were also detected, via their hyperfine splitting of the ground state $\Lambda$ doublet. Biraud et al. $(A A 34,163)$ detected $\mathrm{OH}$ in the $J=3 / 2, F=2-1$ and $1-1$ transitions in absorption before perihelion in 1973 XII. These lines, confirmed by Turner $(A p J 189, \mathrm{L137})$ are the only lines ever found in absorption in a comet. After perihelion Biraud et al. found again $\mathrm{OH}$ but in emission. Mies ( $A p$ $J 189$, L137) and Biraud et al. suggest radiative pumping of $\mathrm{OH}$ to explain the absorptionemission behavior. Black et al. ( $A p J$ 191, L45) detected $\mathrm{CH}$ in the $J=1 / 2, F=1-1$ transition in 1973 XII. Several unidentified emission lines were detected in comet 1973 XII. Giguere and Clark ( $A p J$ 198, 761) found a line at $8.18882 \mathrm{GHz}$ and Buhl et al. (C25) found two lines at 86.2471 and $89.0105 \mathrm{GHz}$.

\section{Negative Results}

Finally, negative results have been reported for the following molecules in $1973 \mathrm{XII}: \mathrm{CH}, \mathrm{CN}$, $\mathrm{CO}, \mathrm{SiO}, \mathrm{H}_{2} \mathrm{O}, \mathrm{HCN}, \mathrm{HNC}, \mathrm{HCO}^{+}$(X-ogen), $\mathrm{NH}_{3}, \mathrm{H}_{2} \mathrm{CO}, \mathrm{HNCO}, \mathrm{HC}_{3} \mathrm{~N}, \mathrm{CH}_{3} \mathrm{OH}, \mathrm{CH}_{2}(\mathrm{CN})_{2}$, $\mathrm{CH}_{3} \mathrm{C}_{2} \mathrm{H}$ and $\left(\mathrm{CH}_{3}\right)_{2} \mathrm{O}$, by Avery and Andrew $(A J 79,1322)$, Buhl et al. (C25) Churchwell et al. ( $A A$ in press), Giguere and Clark $(A p J 198,761)$, Jackson et al. (C25), Schroder et al. ( $A A$ 37,417 ), and Ulich and Conklin (Nature 248, 121). The negative results reported for $\mathrm{OH}$ and HCN are for transitions different than those for which positive results were found. The upper limit for production of $\mathrm{H}_{2} \mathrm{CO}$ reported by Schroder et al. divided by about 100 the upper limit reported earlier by Huebner and Snyder $(A J 75,759)$ for 1970 II. Continuum emissions in the $\mathrm{mm}$ and $\mathrm{cm}$ wavelength ranges have been observed by Hobbs $e$ t al. ( $A p J$, in press) and Bruston et al. (Nature 252, 665). Improvements in resolving power, in pointing accuracy, but mainly in early transmission of accurate ephemerides are needed for better radio observations of comets.

\section{The Vacuum Ultraviolet}

In the vacuum ultraviolet, rocket-borne observations of $1973 \mathrm{XII}$, while detecting again the resonance lines of $\mathrm{H}$ and $\mathrm{O}$, have identified for the first time, that of atomic carbon (Opal $e t$ al., Science 185, 702; Feldman et al., Science 185, 705, also C25; Lillie, C25).

\section{Production Rates}

Bertaux et al. ( $A A 25,415$ ) have given hydrogen production rates in $1970 \mathrm{II}$ and $\mathrm{p} /$ Encke; Keller and Lillie $(A A 34,187) \mathrm{H}$ and $\mathrm{OH}$ production rates in 1970 II. However, 1973 XII provides the most extensive list of production rates for a single comet to date. Mendis and Ip ( $A S S$, in press) give most individual references. Delsemme $(C 20)$ also discusses their weight and their meaning. Reduced to $1 \mathrm{AU}$ by an inverse-square law, in $10^{27}$ particles $\mathrm{s}^{-1}$, he gives $\mathrm{H}(\mathrm{Ly}-\alpha): 25$ to $40 ; \mathrm{H} \alpha:(4) ; \mathrm{OH}, 3090 \AA: 13$ to $15 ; \mathrm{OH}, 18 \mathrm{~cm}:((4$ or 500 ? $))$; O I:26; [O I ] ${ }^{1} \mathrm{D}$ : 11; C I: $6 ; \mathrm{C}_{2}: 2 ; \mathrm{CN}: 0.5 ; \mathrm{H}_{2} \mathrm{O}^{+}((1.0)) ; \mathrm{CH}, 9 \mathrm{~cm}:((300$ ? $)), \mathrm{CH}_{3} \mathrm{CN}(1.0) ; \mathrm{HCN}(2.0)$. None of these results is known better than within a factor of 2 and some are much poorer than that. Results that are uncertain for reasons that are understood are in parentheses; very uncertain, probably much more than an order of magnitude, in double parentheses. The rate determined from $\mathrm{H} \alpha$ (Huppler et $a l ., C 25$ and $A p J 202,276$ ) is uncertain because of the uncertainty of the solar Ly- $\beta$ profile. The identification of water with the most abundant constituent seems more reasonable than ever, since it is consistent with $\mathrm{H}, \mathrm{OH}, \mathrm{OI},[\mathrm{O} \mathrm{I}]$ and $\mathrm{H}_{2} \mathrm{O}^{+}$. Two mechanisms compete to excite O I (1304 $\AA$ ); fluorescence through cascading from O I $\left({ }^{3} \mathrm{D}\right)$, which is excited by coincidence with solar Ly- $\beta$; and resonance scattering, by the chromospheric $O I$ line (By ard and Newson, Nature 249,433). The intensity due to fluorescence is an order of magnitude smaller than that due to resonance, but because of the width of Ly- $\beta$, fluorescence prevails for Doppler shifts larger than $40 \mathrm{~km} \mathrm{~s}^{-1}$. (Feldman et al., C25). The comparison of the rates from $O$ and [O] suggests that the $O\left({ }^{1} \mathrm{D}\right)$ atoms are produced by the decay of a major constituent, possibly $\mathrm{OH}$ itself coming from water. These rates are not different enough either to imply or to reject that there is another source of oxygen atoms not in the ${ }^{1} \mathrm{D}$ state. Only the $\mathrm{OH}$ rates deduced from the ultraviolet band can be accepted, since the radio frequency measurements remain dubious, because the optical depth is not known. The $C_{2}$ and $\mathrm{CN}$ ratios to water seem at least 10 times larger than for p/Encke (Delsemme, Icarus 24, 
95) or for $1970 \mathrm{II}$ (Delsemme, 10.102.011). This seems to be the first numerical evidence of a large difference in the production rate ratios of a 'new' comet like 1973 XII. $\mathrm{CH}_{3} \mathrm{CN}$ and $\mathrm{HCN}$ are the minimum rates associated with an extended source that would just fill up the beam of the radio telescope, but with the assumption of the Bolzmann distribution on the levels involved. The very low population detected on the ground state of $\mathrm{CH}_{3} \mathrm{CN}$ (Buhl et al., C25) suggests that this is not true, and that the minimum value of the production rate could possibly be an order of magnitude lower.

The resonance line of carbon, just because it was obtained with a very poor spatial resolving power, has probably been detected to distances where a large fraction of the carbon compounds had already been dissociated. Since its total rate is compatible with the sum of those of all carbon compounds (at the exclusion of the suspect rate of $\mathrm{CH}$ ), this suggests that the rates of $\mathrm{CH}_{3} \mathrm{CN}$ and $\mathrm{HCN}$ are at any rate not off by an order of magnitude. Brightness profiles across the coma are needed in the light of the atomic resonance lines to yield more accurate production rates.

\section{Brightness Profiles}

The number $g$ of fluorescence cycles per second depends on moderately well known oscillator strength and solar flux values, whereas the lifetime $\tau$ of the emitting molecule results from competitive decay processes that are set by poorly known cross-sections and data from the extreme solar UV. Since the photon flux divided by $g \tau$ yields the production rate, every method to check $\tau$ is welcome; hence the importance of the brightness profiles across the coma, because they yield the actual decay times $\tau$ in space, if the velocity is reasonably known to interpret the decay scale length $s=v \tau$. If the space resolving power is good, they also reveal the extent of their source (parent molecules). Standing in contrast, since one photon [O I] is emitted for each dissociation, the brightness decay of the forbidden $6300 \AA$ line directly yields the production rate and the decay scale of its parent molecule. Delsemme and Combi (BAAS, revised Dec. 1975) give the reduced scale length of [O I] in $1970 \mathrm{II}$ as $10^{5.0 \pm 0.1} \mathrm{~km}$, consistent with $\mathrm{OH}$ as parent. Keller and Lillie $(A A 37,187)$ find indeed $10^{5.1 \pm 0.2} \mathrm{~km}$ for $\mathrm{OH}$ in $1973 \mathrm{XII}$; Blamont and Festou $(C R \quad 278,479)$ had found $10^{5.2+0.2} \mathrm{~km}$ for $\mathrm{OH}$ in $1970 \mathrm{II}$. With this value for the $\mathrm{OH}$ decay, the brightness profiles of $\mathrm{OH}$ in $1960 \mathrm{II}$ (Delsemme, C5), $1970 \mathrm{II}$ (Keller et al., $A A 34,87$ ), and 1973 XII (Blamont et al., $C R 278,479$ ) all yield a scale length of $10^{5.0 \pm 0.1}$ for the $\mathrm{OH}$ parent, which is consistent with its identification with water (Delsemme, C25). The scale length for hydrogen ionization has been established from the Ly- $\alpha$ isophotes (Bertaux et al., $A A$ 25, 415; Keller, $A A$ 27, 51).

Delsemme (C25) also computes, for 12 previously published brightness profiles of $\mathrm{CN}$ and 14 of $\mathrm{C}_{2}$, the scale length for radical production $s^{\prime}$ (from the parents' assumed dissociation) and that for radical decay $s$ (by further dissociation or ionization). Observed profiles do not distinguish between different models in the $10^{4} \mathrm{~km}$ range, therefore $s^{\prime}$ yields the crude extent of the source function, but not its actual distribution. However, Delsemme's data on 8 different comets, which confirm Delsemme and Moreau's ( $A L$ 14, 181) results for 1970 II, remain consistent with $s=s_{0} r^{2}$ and $s^{\prime}=s_{0}^{\prime} r$, with $\log s_{0}=4.82$ for $\mathrm{C}_{2}$ and 4.1 for $\mathrm{CN}$ ( $s$ and $s^{\prime}$ in $\mathrm{km}, r$ heliocentric distance in $\mathrm{AU})$. The observed values of $s(\mathrm{CN})$ and $s\left(\mathrm{C}_{2}\right)$ as well as their dependence on the square of the distance, are both consistent with a decay induced by the solar flux only. However, the approximate proportional dependence on distance for $s^{\prime}$, suggested by both $\mathrm{C}_{2}$ and $\mathrm{CN}$ profiles, is inconsistent with photodissociation, whereas this dependence had been predicted by Delsemme and Miller's model of an icy-grain halo surrounding the nucleus. Probable detection of the icy-grain halo is reported by Hobbs et al. (see Section D) in 1973 XII.

\section{The Hydrogen Coma}

Improving on Keller's (09.102.003) multiple scattering model of the $\mathbf{H}$ coma of $1970 \mathrm{II}$, Keller and Thomas $(A A 33,7)$ determine an absolute Ly- $\alpha$ flux of the Sun, from the geometry and the distortion of the isophotes. They require a bimodal distribution of $\mathrm{H}$ velocities with 7 and $21 \mathrm{~km} \mathrm{~s}^{-1}$ peaks, conceivably justified by the dual origin of the $\mathrm{H}$ atoms, since the dissociation of $\mathrm{OH}$ probably has a different energy balance from that of $\mathrm{H}_{2} \mathrm{O}$. However, Keller et al. $(A A 38,413)$ report that the width of the Ly- $\alpha$ line, observed by Skylab in the inner coma of 
$1973 \mathrm{XII}$, is consistent with a velocity of 8 to $10 \mathrm{~km} \mathrm{~s}^{-1}$. Conceivably, the large velocity component is hidden in the wing of the line profile, because of its weak contribution to the light of the inner coma. Emission line profiles are predicted by Meier $(A A 40,373)$ for Ly- $\alpha$ and O I (1304 $\AA$ ).

VIII. ${ }^{12} \mathrm{C} /{ }^{13} \mathrm{C}$ Isotopic Ratio

The ${ }^{12} \mathrm{C} /{ }^{13} \mathrm{C}$ isotopic ratio had been measured only once, from the $\mathrm{C}_{2}$ Swan system in $1963 \mathrm{I}$ by Greenstein and Stawikovski. Now three more measures have been reported by the same technique; in 1969 IX by Owen ( $A p J 184,33)$; in 1973 XII by Danks et al. $(A p J 194,745)$ and in $1975 \mathrm{~h}$ by Vanysek (unpublished). The results remain very uncertain $( \pm 50 \%)$ because of the blend with the $\mathrm{NH}_{3}$ bands, but within these limits no inconsistencies with the terrestrial ratio have appeared in any of the four comets.

\section{Pure Photochemistry}

Pure photochemistry can be observed in the cometary exosphere $\left(>10^{5} \mathrm{~km}\right.$, no collisions). Therefore observed large velocities, in particular for $\mathrm{H}$ (Bertaux et al., $A A$ 25, 415; Keller, $A A$ 27, 51), for $\mathrm{OH}$ (Biraud et al., $A A 34,163$ ), for CH (Black et al., ApJ 191, L45), probably result from the energy balance of the dissociations, and should therefore be more and more used for identifying parent molecules. Jackson ( $C 25$ and J. Photochem., in press) suggests that $\mathrm{NH}, \mathrm{C}_{2}$ and $\mathrm{C}_{3}$ are produced in comets by photolysis of $\mathrm{NH}_{4}, \mathrm{C}_{2} \mathrm{H}_{2}$ and $\mathrm{C}_{3} \mathrm{H}_{4}$, with $\mathrm{NH}_{2}, \mathrm{C}_{2} \mathrm{H}$ and $\mathrm{C}_{3} \mathrm{H}_{2}$ as intermediate steps. High-dispersion spectra of $1970 \mathrm{II}$ have confirmed (Arpigny, $C 25)$ the usual assumption of a small expansion velocity of $1.0 \pm 0.3 \mathrm{~km} \mathrm{~s}^{-1}$ for the $C_{2}$ and $C N$ coma.

\section{The Cometary Ionosphere}

\section{I. $\mathrm{H}_{2} \mathrm{O}^{+}$}

The tentative identification (Herzberg and Lew, $A A 31,123$ ) of the $\mathrm{H}_{2} \mathrm{O}^{+}$bands in the optical spectrum of 1973 XII, obtained by Benvenuti and Wurm $(A A 31,121)$ has been confirmed by Wehinger et al. (ApJ 190, L43) from medium and large dispersion spectra, obtained at both Wise and Lick observatories and showing nearly 50 identifications. A few of these unidentified bands had been reported in $1942 \mathrm{~g}$ and $1963 \mathrm{I}$ and are also probably visible in $1941 \mathrm{I}$, in Swings and Haser's Atlas of Cometary Spectra. Wehinger and Wyckoff (ApJ 192, L41) report their presence in 1974b; Delsemme and Combi (BAAS, Dec. 1975) in 1970 II. The $\mathrm{H}_{2} \mathrm{O}^{+}$ion completes the identification of the major photodissociation and photoionization products of $\mathrm{H}_{2} \mathrm{O}$, whose neutral molecule was also identified, a few weeks later, in $1974 \mathrm{~b}$.

\section{Ion Sources in Inner Coma}

The origin of the ionic species, although still in doubt, may soon be elucidated. The longstanding argument over the place and mechanisms of their formation, has been superseded by proposals for fast ion-molecule chain reactions in the inner coma (probably initiated by photoionization only) and that could well be Wurm's 'intrinsic cometary processes.' Cherednichenko has been the pioneer of these ideas. More recently, he has again (10.102.28) emphasized their importance to form $\mathrm{CO}^{+}$and $\mathrm{N}_{2}{ }^{+}$; he gives a formula to assess their cross-sections. Wurm's morphological arguments requesting short length and time scales, developed again (11.102.005) from the Helwan plates of $\mathrm{p} /$ Halley, may probably be understood in terms of the small size $\left(\leq 10^{4} \mathrm{~km}\right)$ of the collisional zone in the inner coma, since the need of collisions is obvious for the propagation of these fast chain reactions. Since many ion-molecule reactions have no activation energy, their rate is almost temperature independent. Meot-Ner and Field (Origins of Life 6, 377) even report negative temperature dependences. More recently, Oppenheimer ( $A p J$ 196, 251 and $\mathrm{C} 25$ ) lists some 100 reactions, half of them involving ions, that could reshuffle the molecular species of the inner coma, but he renounces to predict a steady state model. Rough models of the ionosphere of the $\mathrm{H}_{2} \mathrm{O}$ coma are however already developed; Aikin (12.102.011) emphasizes the role of the $\mathrm{H}_{3} \mathrm{O}^{+}$ion that in his model predominates in the inner coma. Biermann and Diercksen (12.102.017) also mention its importance. The spectrum of 
$\mathrm{H}_{3} \mathrm{O}^{+}$is unknown and therefore will remain unobserved in comets for some time. However, if water is indeed a major constituent, it is worth mentioning here that most ions containing hydrogen will be converted fast into $\mathrm{H}_{3} \mathrm{O}^{+}$by exchanging a proton with $\mathrm{H}_{2} \mathrm{O}$. Other coma models incorporate photo-ionization with photoelectron cooling, ion-molecule interactions and dissociative recombinations (Wallis and Ong, C25; Shimizu, $A S S$, in press). Altogether, the ion density is found to be small and the plasma pressure low. This is rather confirmed by the production rates of $\mathrm{H}_{2} \mathrm{O}^{+}$, deduced from observations by Wyckoff and Wehinger $(A p J$, in press) for 1973 XII, and by Delsemme and Combi (BAAS, Dec. 1975) for $1970 \mathrm{II}$. However, Ip and Mendis (Icarus in press) claim that dissipating of hydromagnetic waves could be a significant source of heating, and that large electric currents could be the most important source of ionization in the coma.

\section{Interaction with Solar Wind}

Standing in contrast with the collisionless models of the outer neutral coma, the magnetic collision of the ionized coma with the solar wind must still be described by a fluid dynamics approach. Schmidt (C25) reports low-shock Mach numbers close to $t w o$, in his axi-symmetric model of the solar-wind flow through the outer coma, with source terms representing ionization effects. Wallis (10.102.016) applying this type of model to an $\mathrm{H}_{2} \mathrm{O}$ coma the size of $1970 \mathrm{II}$, finds the bow-shock at 2-3 million $\mathrm{km}$ ahead of the nucleus. He suggests with Ong (C25) that the assumed 'contact surface' between solar wind and cometary plasma is not real, in view of the low ionospheric pressure and stagnation pressure. The smoothness of the $\mathrm{H}_{2} \mathrm{O}^{+}$sun wards brightness profile in 1970 II (Delsemme and Combi, BAAS, Dec. 1975) does not suggest the existence of a sharp transition to solar wind flow before $10^{5} \mathrm{~km}$. An internally-generated cometary magnetic field has been hypothesized by Mendis and Alfvén (11.102.014) to be associated with plasma structure inside the contact surface. Small intrinsic magnetic fields in the nucleus or the coma could be amplified by turbulent motions. Ioffe $(10.102 .029)$ and Schmidt (1974) have pursued the idea of an enhanced solar wind field.

\section{E. The Dusty Coma}

The need of a separate description of the dusty coma, arises from differences in the detection techniques, since the high concentration of dust in the center of the head allows its easy detection as a continuum in optical spectra as well as in infrared, whereas the distribution in the tail is usually studied from photographs in total light and from kinematics considerations.

\section{Origin of Dust}

Although most authors assume that the particles leaving the coma originate in the nucleus and are dragged away by vaporizing gases, Dolginov (08.102.027) argues that the molecular abundances, densities and temperatures of the inner coma would allow the formation of these particles outside of the nucleus.

\section{Dust Emission Features in Infrared}

Ney $(A p J 189, \mathrm{~L} 141$ and Icarus 23,551$)$ has detected again in infrared, two broad but rather typical emission features near 10 and $18 \mu \mathrm{m}$ superimposed on the thermal continuum, that probably came from the infrared emission spectrum of small silicate grains. This silicate 'signature,' first observed in 1970 II, was also present in 1973 XII and 1974b. The rest of the thermal continuum fits rather well a black-body curve between 2 and $20 \mu \mathrm{m}$, whose temperature varies with heliocentric distance between $350^{\circ}$ and $700^{\circ} \mathrm{C}$, yielding a dust albedo of the order of 0.2 . Rieke and Lee (Nature 248, 737) and Rieke et al. (C2) also detect the silicate signature, but they conclude from their study of the infrared spectra of 1973 XII and other comets that, while silicates are the only material probably identified in cometary dust, the composition of the dust varies from comet to comet and also with time for any particular comet. 1973 XII was also measured in the infrared by Gatley et al. (Icarus 23, 561) and by others, confirming the $10 \mu \mathrm{m}$ silicate signature and a particle color temperature higher than a 
black-body temperature. From thermal emission variations of 1965 VIII versus heliocentric distance, Shul'man (12.102.12) shows that the dust grains were semiconductive.

III. Sunlight Scattered by Dust

Stokes $(09.103 .100)$ finds distinct reddening by spectrophotometry of $1970 \mathrm{II}$ (4500-8000 $\AA$ ) and concludes that the most important scattering particles were larger than $0.1 \mu \mathrm{m}$. Babu and Saxena (09.103.100) conclude from their spectrum scans $(3520-6120 \AA)$ over a range of phase angles, that the particles are about $0.54 \mu \mathrm{m}$. O'Dell (1974a) combines several optical studies of $1970 \mathrm{II}$, together with infrared observations, to link particle density and size with radiation pressure scattering efficiency. The reddening of scattered light of 1970 II decreased as the phase angle increased (Babu, C5).

\section{Fluid Dynamics of the Dusty Coma}

Reported gas production rates do not leave much room for doubt any more, that there is a rather large collisional zone near the nucleus. Hence hydrodynamic descriptions, in the FinsonProbstein style, are used for investigating transsonic flow in the inner coma, but heat sources must be introduced. For instance the dust absorbs radiation and may heat the gas (Shul'man, 1972). Temperature derived from the Greenstein effect (Arpigny, C25) rather support such a heating, although Shimizu (C25) argues that rotational excitations should rapidly cool the gas. Wallis (11.102.001) gives an analytic solution for an $\mathrm{H}_{2} \mathrm{O}$ coma, with heating from photodissociations, in which the radial velocity and temperature increase outwards, unlike adiabatic solutions. The transition from hydrodynamic gas flow to effusion in vacuum is still being discussed (Wallis, 12.102.010) but no good description is yet available. In particular, the multifluid approach (Ip and Mendis, 11.102.002) describes rather well how the radial velocities of the different gases can separate in the transition region $\left(10^{3}\right.$ to $\left.10^{5} \mathrm{~km}\right)$, but 'ad hoc' polytrope indices artificially simulate the heating. This approach seems at least to explain the low velocity $\left(8 \mathrm{~km} \mathrm{~s}^{-1}\right)$ of the expanding hydrogen; the same authors also examine the effects of gas released by an extended halo of vaporizing icy grains.

\section{Halo of Icy Grains}

Probable detection of such a halo has been reported by Hobbs et al. (ApJ in press) in 1973 III by its emission continuum at $3.71 \mathrm{~cm}$.

\section{F. Production Laws of Gas and Dust}

\section{Wide-Band Brightness Laws}

Visual or $U B V$ light curves are still being steadily published. Unfortunately, since these curves do not separate either the individual molecular bands or the dust, their use is limited, either to the description of the irregular activity of many comets (Jacchia, 11.102.003), as exemplified by Morris and Bortle $(P A S P$ 85, 249) for $1968 \mathrm{I}$; Marsden $(Q J R A S 15,433)$ for $1973 \mathrm{VI}$; Angione et al. (Icarus 24, 111), Tanabe (Tokyo Astron. Bull. 235, 2719), Kiselev et al. (KTK 164), Kohoutek et al. (C25) and Isserstedt et al. $(A A$ 41, 9) for 1973 XII; Rosenbush (KTK 173) for 1974b; or to the study of secular trends, as the weakening of P/SchwassmannWachmann I and P/Oterma (Vsekhsvjatsky, $P K F$ 9,110). Wide acceptance of a system of special photometric filters for cometary work, separating the lights from the different molecular emissions and the dust, is still much needed.

\section{II. . Monochromatic Brightness Laws and Production Laws}

When observed through a finite diaphragm (or when limited by the sky background) the brightness dependence on distance in the light of a radical is not the same as that of the production rate of that radical. The two laws are connected theoretically for the gas coma by Delsemme (10.102.001). Applying his theory to $1969 \mathrm{IX}$, he shows first that the brightness law in $r^{-6}$ observed for $\mathrm{OH}$ and for $\mathrm{H}$ by the OAO corresponds to a production law in $r^{-2.8 \pm 0.2}$, consistent with the vaporization of water snow at that distance (the exponent grows with distance), but inconsistent with a stuff much more volatile than water. Next, a careful model of 
the $\mathrm{H}$ and $\mathrm{OH}$ coma of $1970 \mathrm{II}$ by Keller and Lillie $(A A 34,187)$ yields $r^{-2.3 \pm 0.3}$ for both production laws, which is also consistent with water at this distance. Finally, another careful model of the coma of 1973 XII (A'Hearn and Cowan, $A J$ 80, 852) yields the same production law in $r^{-3.5 \pm 0.5}$ for $\mathrm{CN}$ and for $\mathrm{C}_{2}$, which is not inconsistent with a vaporization controlled most of the time by water, but which was perturbed on 1973, December 1 and 2 by a large outburst observed in $\mathrm{C}_{2}$ light. Huppler et al. (C25) also mention that in $1973 \mathrm{XII}, \mathrm{H} \alpha$ and [O I], observed with a Fabry-Pérot, vary as $r^{-3.3 \pm 0.4}$ (uncorrected brightness law).

\section{Outbursts}

Outbursts often perturb the steady state assumed in theoretical brightness laws or brightness profiles. Tarashchuk et al. ( $A A K 28$ ) report an outburst of 1973 XII that produced striking changes in both absolute and relative emission rates observed in spectral scans, in a time scale of several minutes. Borisov (KTK 156), Afanasjev et al. (KTK 151 and 159), and Simonenko ( $K T K$ 156) used the same technique. Cernych $(K T K 162,172)$ detected an outburst of P/Schwassmann-Wachmann I in August 1974; Riives (Tartu, in press) analyzed the February outburst of 1969 IX: the observed halo expanded at $1.1 \mathrm{~km} \mathrm{~s}^{-1}$, its tailward acceleration was $0.66 \mathrm{~cm} \mathrm{~s}^{-2}$. Kresak (BAIC 25, 293) discusses the 1973 nine-magnitude outbursts of $\mathrm{p} /$ TuttleGiacobini-Kresak; these are the largest outbursts ever reported. The production law of hydrogen from 1973 XII also showed large irregularities in the Ly- $\alpha$ light, observed from Skylab near perihelion, and from a rocket (Carruthers et al., Icarus 23, 526 and $A A$, in press). Despite its smaller reduced brightness, 1969 IX had a larger H production rate than 1970 II. Keller (C25) explains it by a smaller dust output. Isserstedt and Schlosser $(A A 41,9)$ correlate intensity fluctuations of 1973 XII with oscillations of the solar plasma.

\section{Outburst Mechanisms}

Outburst mechanisms are tentatively explained by Shul'man ( $A A K 24,91)$ by radiative chemical processes, which justifies why they occur mainly at large heliocentric distances. Kresak (BAIC 25, 293) believes that the largest outbursts ever observed can be best explained by rotational breakup. The transition from amorphous to cubic ice, proposed by Patashnick $e t$ al. (12.102.001) does not seem very promising as an energy source, because impurities stop the formation of amorphous ice (Delsemme, C20). The mere number of different mechanisms proposed during the last decade suggests that the triggering of outbursts has not yet been elucidated.

\section{Optical Spectrophotometry}

Direct optical spectrophotometry of the central coma of 1973 XII, yields absolute ratios for the continuum and many molecular bands. It was used by A'Hearn $(A J 80,861)$ with a Fourier-transform spectrometer, and by Tarashchuck et al. (AAK 28), who report fast variations in the intensity ratios. This technique could become fundamental in establishing brightness laws. However, their dependence on a diaphragm factor (Delsemme 10.102.001) implies that caution should be used in deducing production laws. Narrow pass-band photometry with interference filters (Simonenko, KTK 171, Rozhkovsky and Gorodetsky, KTK 164) or from objective prism photographs (Kurchakov and Gorodetsky, C8; Beitrishvili, KTK 165) or in photographic IR (Kim Gun Der and Makarov, KTK 161) were also used from 1973 XII and 1974b. Objective-prism photographs of 1963 III do not show any change after its brightening (Grudsinska, Acta Astron., in press).

\section{G. Dust Tails}

\section{Dust Kinematics and Dynamics}

No fundamental distinction has ever emerged from the morphological differences between type II and type III tails, and it is now felt more and more that both can be explained by dust dynamics. The traditional analysis in terms of synchrones and syndynames has given way to a more sophisticated approach, where a continuous emission at varying rates of a dust distribution of varying sizes, can be deduced from the tail isophotes. The larger number of para- 
meters is partially compensated by the fact that the initial velocity of the grain is no more a free parameter. It is identified with that terminal velocity of the grain when it emerges from the inner coma, which is the result of the fluid-dynamics interaction of gas and dust in the collision zone: the larger the grain, the smaller its velocity, which goes down from the gas-velocity value to zero. This approach, first described by Finson and Probstein and convincingly applied to 1957 III, has been further applied successfully to 1970 II and 1962 III.

For 1970 II, Sekanina and Miller (Science 79,565) find subkilometer-per-second particle emission velocites, particle sizes of typically a few $\mu \mathrm{m}$, a dust production rate of $2 \times 10^{7} \mathrm{~g} \mathrm{~s}^{-1}$ at $0.56 \mathrm{AU}$ from the Sun and a radius of $2.6 \mathrm{~km}$ for the comet's nucleus. Konopleva et al. ( $A A K 22,61$ and 27) compute the acceleration of dust in 1970 II and 1973 XII. The cutoff of the particle size distribution in 1970 II is discussed by O'Dell (Icarus 21, 96). For 1962 III, Jambor $(A p J 185,727)$ obtains somewhat lower emission velocities and larger particle sizes, and find that a sharp variation in the production rate of dust at perihelion is the cause of the observed 'split' tail (or spurious 'shadow' of the nucleus). Whereas the Finson-Probstein's approach is almost universally accepted, Wurm $(A S S 27,211)$ points out that its fundamental assumption of isotropy in the dust emission is incompatible with the frequent presence of sun ward dust streamers in the inner coma of many comets. Benvenuti $(A S S 27,203)$ finds that the dust structures near the nucleus vary from comet to comet.

\section{Sunward Tails}

If early emission times of large (millimeter-size) particles are assumed (Sekanina, 11.102.021), the appearance of a sunward tail (antitail) becomes only a matter of geometry that can be easily predicted. Sekanina (C31) has checked that 20 to $30 \%$ of nearly-parabolic comets, for which the geometry is favorable, do display an antitail, whereas (virtually) no short-period comets do so. This suggests that only comets rather recently captured from Oort's cloud, vaporize gases that are volatile enough to drag away large particles at large heliocentric distance.

As predicted earlier by Sekanina (11.102.021), the sunward tail of 1973 XII was observed shortly after perihelion from Skylab III both visually (Gibson, Icarus 23,493) and photographically (MacQueen $e t$ al., $C 1$ ) and from the ground in the infrared (Ney, $A p J 189$, L14l and Icarus 23, 551). From the non-detection of the silicate signature at $10 \mu \mathrm{m}$ in this antitail, Ney concludes that its particles are larger than $10 \mu \mathrm{m}$. Physico-dynamical models of this antitail are proposed by Sekanina (Icarus 23, 502), who uses the Finson-Probstein approach with negligibly small emission velocities; by Gary and O'Dell (Icarus 23, 519), who applied a simplified syndyne approach; and by Lamy and Koutchmy (C31). Sekanina, as well as Gary and $O$ 'Dell find that large particles are present, the estimates ranging from a few tens $\mu \mathrm{m}$ - to mm-sized grains, whereas Lamy and Koutchmy suggest a possibility of very tiny dust particles, typically $0.02 \mu \mathrm{m}$ in radius, in order to explain the strongly polarized light of the antitail in mid-January 1974 (Bucher et al., AA 39, 289).

\section{Vaporizing Particles}

Sekanina and Miller (Icarus, in press) compare Sekanina's model with absolute photometric profiles from the Cerro Tololo plates of 1973 XII and confirm the evidence for vaporizing dust particles. Spinrad and Smith (Icarus 19,419) explain a $7000 \mathrm{~km}$ sunward offset between the peak of the sodium emission, and that of the continuum of 1970 II, by a vaporization of dust grains. Sekanina $(10.102 .005$; Icarus 25,218$)$ explains several of the unexplained features of the tails of some distant comets (perihelia beyond $2.2 \mathrm{AU}$ ) assuming that they are made of rather large $(\geq 0.1 \mathrm{~mm}$ ) grains of water ice (or of solid hydrates). These grains are apparently emitted (anisotropically) from the nucleus at distances up to $15 \mathrm{AU}$ from the Sun, so that a substance much more volatile than water snow (at least as volatile as $\mathrm{CO}_{2}$ ) must be present in the nucleus of these comets, in order to provide the necessary gas drag for grain ejection at these very large distances. These results are in agreement with the fast decay of new comets, after their first passage at short heliocentric distances. In any future approach, Patashnick and Rupprecht's ( $A p J 197$, L79) results should be taken into account, since they showed that the sublimation rate of icy grains depends considerably on their size, with a deep minimum at $50 \mu \mathrm{m}$ around $1 \mathrm{AU}$. 


\section{Tail Polarization}

Weinberg and Beeson (C25) found a very strong negative polarization of the light in the tail of 1965 VIII, interpreted in terms of slightly absorbing submicron dust particles of a very narrow size distribution. Polarimetry of 1973 XII in January 1974 (Bugaenko et al., KTK 167) yields particles $>0.4 \mu \mathrm{m}$ with a complex index of refraction $\mathrm{m}=1.2-2.3 \mathrm{i}$ (Shul'man $A A K$, in press).

\section{Dust Detection in Space}

Small dust particles (for which radiation pressure balances solar gravity) were reported to have been detected by the Earth-orbiting satellite HEOS 2 (Hoffmann et al., C25; Grun et al., C31). An enhanced flux of $10^{-13}$ to $10^{-11} \mathrm{~g}$ particles, moving at 15 to $20 \mathrm{~km} \mathrm{~s}^{-1}$, observed from mid-May until early August 1974, was apparently due to emissions from 1973 XII at solar distances near or beyond $4 \mathrm{AU}$.

\section{H. Plasma Tails}

\section{Kinematics}

Miller $(A J 78,1126)$ describes the development and motions of rays and filaments in the plasma tail of 1957 III on May 5, 1957, and compares with Wurm's phenomenological description of $1908 \mathrm{III}, 1960 \mathrm{II}$ and 1970 II. Noticeable changes take place in $1.5 \times 10^{3} \mathrm{~s}$, but no evidence for ray-closing is observed. Velocities of tail structures range from 10 to $300 \mathrm{~km} \mathrm{~s}^{-1}$ in $1970 \mathrm{II}$, and a slight correlation is suggested between kinks in the tail axis and changes in the direction of the solar wind (Jockers and Lüst, $A A 26,113$ ). The tail features and their motions are beautifully shown in a motion picture of 1973 XII by Anzer, Jockers and Schmidt (10.103.102). Tail structure may have been observed in radio-scintillation observations of the radio-source PKS 2025-13 during the passage of 1973 XII, although details of the interpretation are not clear (Ananthakrishnan et al., ASS, in press).

\section{Dynamics}

The question of wave versus material motion of tail features may be difficult to solve without a direct test through observed Doppler shifts. Jockers et al. (C25) interpret the tail velocities of 1973 XII in terms of material motions; whereas Hyder et al. (12.103.104) who measure velocities of $250 \mathrm{~km} \mathrm{~s}^{-1}$ in $1973 \mathrm{XII}$, interpret many of the moving features (in particular on January 13, 1974) as waves propagating at the Alfvén speed; they suggest that the observed helical structure might be triggered by the kink plasma instability; however, Ershkovich (PSS, in press) suggests an origin via the Kelvin-Helmholtz instability, which seems more likely. A discussion of plasma tail shapes by Brandt and Rothe (C25) expects some curvature on the basis of the wind-sock theory applied to the entire tail. For a constant solar wind, the curvature depends on only one parameter, function of orbital and solar-wind conditions. Chernikov (12.102.002) proposes a mechanism of momentum transfer from the solar wind to the cometary plasma.

\section{Tails as Solar-Wind Probes}

Brandt, Harrington and Rosen (10.106.014 and $A p J 196,877)$ use a $75-y r$ sample of plasmatail observations to derive mean solar-wind properties: radial speed $402 \mathrm{~km} \mathrm{~s}^{-1}$; azimuthal speed $7 \mathrm{~km} \mathrm{~s}^{-1}$; maximum meridional speed $2.6 \mathrm{~km} \mathrm{~s}^{-1}$ (flow diverging away from solar equator). They find no evidence of solar-wind speeds changing with latitude in contrast with Tarashchuk (09.102.025) who presents such evidence. This question is discussed again by Andrienko $(P K F 9,104)$ and Tarashchuk $(A A K 21,62 ; 22,53 ; 22,57)$.

\section{Coupling between Ion Tail and Solar Wind}

Hyder et al. (12.103.103) and Ip and Mendis (ASS 35, L1) have proposed currents along the comet tail and associated helical field, to explain certain visible kinks and radio scintillations. Ershkovich (IAGA) has alternatively developed earlier work with Chernikov (09.102.007) to explain the screw-features as large amplitude MHD helical waves. The detailed coupling mechanism between solar wind and comet tail plasma has been studied further. Chernikov 
(12.102.002) puts it down to ion-sound turbulence driven by relative streaming velocities, while Wallis and Ong (1975) find support in recent non-linear plasma theory for the previous inhomogeneous shearing flow model with transverse wave turbulence.

\section{Laboratory Studies Relevant to Comets}

\section{Spectroscopy}

The most important work was the observation and detailed analysis of a laboratory spectrum of $\mathrm{H}_{2} \mathrm{O}^{+}$by Lew and Heiber $(J C P 58,1246)$ that lead to the first identification of a new cometary ion since 1942 (see Section D). Because of the large number of emission lines of $\mathrm{H}_{2} \mathrm{O}^{+}$observed in the laboratory, the detailed analysis of this spectrum was a prerequisite for the identification of the cometary features which correspond to very low temperature. With the increasing availability of vacuum UV spectra of comets, it is important to have complete data available about various diatomic and polyatomic molecules. Diatomic molecular spectra are still being studied in Ottawa, in particular those of the $\mathrm{H}_{2}$ molecule and its isotopes (Bredohl and Herzberg, CJP 51, 867; and Dabrowski and Herzberg, CJP 52,1110 and in press). The vacuum UV spectrum of $\mathrm{OH}$ has been reported by Douglas $(C J P 52,318)$. A spectrum of $\mathrm{C}_{2}{ }^{+}$has been obtained in absorption (Meinel, CJP 50,158). A spectrum near $2300 \AA$, probably due to $\mathrm{NH}_{2}{ }^{+}$, has been observed in the laboratory by Herzberg (unpublished). The predissociation observed in the vacuum UV spectrum of $\mathrm{H}_{2} \mathrm{O}$ might be responsible for the inversion of the ground state that leads to the $\mathrm{H}_{2} \mathrm{O}$ laser emission in interstellar clouds (Oka, 11.012.025). The same mechanism may apply to the observed $\mathrm{H}_{2} \mathrm{O}$ emission in $1974 \mathrm{~b}$.

\section{Radiative Lifetimes}

Radiative lifetimes of cometary ions and molecules in their excited levels, are still much in demand to compute the fluorescence steady state and deduce cometary densities and production rates. Erman and Brzozowski's (Phys. Lett. 46A, 79) laboratory lifetimes for the ${ }^{2} A_{1}$ excited state in $\mathrm{H}_{2} \mathrm{O}^{+}$were used for this purpose in 1970 II and 1973 XII (Section D). Curtis et al (Physica Scripta, in press) find oscillator strengths for the Swan bands of $\mathrm{C}_{2}$, that are $40 \%$ lower than previous estimates. UV photoabsorption data, including ionization or dissociation rates, are still missing or insufficient to compute space lifetimes of many molecules ( $\mathrm{HCN}, \mathrm{CH}_{3} \mathrm{CN}$ and possible parents like $\mathrm{HC}_{2} \mathrm{CN}, \mathrm{C}_{3} \mathrm{H}_{4}$ etc.) as well as for most ions and radicals observed in comets. More accurate data on the solar flux in the vacuum and extreme UV are expected soon from the Naval Research Laboratory group in Washington, D.C.

\section{Collisional Excitation}

In Leningrad, emission spectra induced by electron bombardment of organic vapors, like $\mathrm{CH}_{3} \mathrm{CN}$ or amino-acids, are reported by Kaimakov and Matveev (in press). The $\mathrm{C}_{2}$ emission appeared only when the original organic substance had more than five $\mathrm{C}$ atoms. Cross-sections for dissociative excitation by electrons of a variety of simple organic substances are studied by Sushanin $(P K F 8,85)$ and Sushanin and Koshko $(A Z 51$, No. 6, 447).

\section{Laboratory Simulation of the Icy Conglomerate}

Kaimakov et al. (in press) study the sublimation of icy mixtures and the outflow of dust under the influence of $10-60 \mathrm{eV}$ electrons or $0.5-4.0 \mathrm{eV}$ protons. The electrons increase, and the protons decrease the velocity observed for neutral grains. During the sublimation of frozen solutions of $\mathrm{CH}_{3} \mathrm{CN}$ in water, formation and outflow of icy dust was observed by Kaimakov and Aliev ( $D A T$ 18, No. 4, 16). High density amorphous ice was not confirmed (Kaimakov et $a l$, in press). However, recent X-ray data (Wenzel et al., Science 187, 430; Narten et al., unpublished) show that there are two forms of amorphous solid water with densities 0.93 and $1.2 \mathrm{~g} \mathrm{~cm}^{-3}$. Delsemme believes now that the $1.2 \mathrm{~g} \mathrm{~cm}^{-3}$ form of amorphous ice was already observed in 1970 by Delsemme and Wenger, but that its density was reported too high (C20). 


\section{J. Comets and Space Research}

I. Ballistic Fly by $p /$ Encke in 1980

Encouraged by resolution No. 7 of the IAU (Sydney 1973), efforts to define a first interplanetary mission to a comet seem to have concentrated on a fly-by of $p /$ Encke in 1980 . In Europe, a study of a $16 \mathrm{~km} \mathrm{~s}^{-1}$ fly-by, with a modification of the Helios spacecraft seems to request more effort to approach realization. In the United States, NASA's 1975 Comet Working group finds that the best set of available missions for the next decade is a mission to p/Encke in 1980 with encounter at a heliocentric distance near $0.4 \mathrm{AU}$; and a single launch of two identical spacecrafts in 1985, one targeted for a $p /$ Halley fly-by and the second placed on multiple encounter trajectory to $\mathrm{p} /$ Giaccobini-Zinner and $\mathrm{p} /$ Borelly. The proposed payload for the first mission requires imaging the cometary nucleus, studying the sources of the cometary atmosphere, determining the structure, dynamics and composition of the coma, measuring dust, fields, and energetic particles; the spacecraft would fly through the inner coma; its minimum distance could typically be $500 \pm 300 \mathrm{~km}$ from the nucleus and the fly-by velocity $13 \mathrm{~km} \mathrm{~s}^{-1}$. The U.S. Space Science Board (Opportunities and Choices in Space Sciences, 1974, N.A.S., Washington, D.C. 1975) has backed this ballistic fly-by of p/Encke in 1980, which may be done with a spare Mariner-Venus-Mercury spacecraft. Because the physical study of comets is of a fundamental importance in unraveling the cosmogony of the solar system, Commission 15 urges that NASA considers this mission as one of its key new programs.

\section{Space Telescopes}

The recent discovery of the resonance lines of $\mathrm{H}, \mathrm{C}$ and $\mathrm{O}$ in 1973 XII (see Section $\mathrm{C}$ ) and Delsemme's $(C 20)$ proposal to use the ratio of these elements' production rates as a cosmothermometer of the accretion temperature of comets in the primeval nebula, emphasizes the importance of observing comets in the vacuum UV. New space telescopes will be available soon for this purpose, in particular the International Space Explorer, a $45-\mathrm{cm}$ space telescope with a large echelle grating spectrograph, an international undertaking sponsored by NASA and ESRO, to be launched in 1977. It is recommended that brightness profiles of the coma of bright comets be measured in the resonance lines of the major elements.

\section{Zero-Gravity Studies}

Zero-gravity studies of cometary snows and of their accretion properties are being discussed at NASA's Marshall Space Flight Center, in connection with a proposal to use Spacelab facilities in the 1980 ies.

\section{MINOR PLANETS}

\section{The Physical Study of Minor Planets}

The physical study of minor planets was promoted as a discipline in 1971 at C12 (NASA-SP 267, ed. Gehrels) and appears in the IAU report for the first time. Positions and motions, including search and discovery, are still reported by Commission 20.

\section{Masses and Densities}

The only mass measured gravitationally (from Arete's perturbations, by Hertz in 1966) was that of Vesta $\left(1.20 \pm 0.12\right.$ in $10^{-10}$ solar units). Now, Schubart $(A A 30,289 ; A A 39,147)$ has determined the mass of Ceres $(5.9 \pm 0.3)$ and Pallas $(1.14 \pm 0.22)$ from their mutual perturbations. Combined with the new diameters deduced from albedos, these masses yield densities of $2.6 \mathrm{gm} \mathrm{cm}^{-3}$ for Ceres, 2.5 for Pallas and 3.6 for Vesta ( \pm 15 or $20 \%$ ).

\section{Albedos}

In principle, the cross-sectional area $S$ and geometric albedo $A$ can be separated by assessing the reflected flux AS by visible photometry, and the absorbed flux $(1-\mathrm{A}) \mathrm{S}$ at steady state by the amount of energy radiated back to space in the infrared (radiometry from 10 to $20 \mu \mathrm{m}$ ). 
Following early measurements by Allen (03.098.036) and Matson (07.098.036), sources of uncertainty were discussed by Morrison (09.091.057) and Jones and Morrison (12.098.010). Morrison (12.098.062) gives the results for 40 asteroids, Morrison and Chapman for 22 ( $A p J$, in press), and Hansen for $84(A J 81,74)$. Cruikshank (Icarus, in press) measured 4 Trojans, all of low albedo. An alternative and equally productive means of determining albedos from the slope of a plot of linear polarization against phase angle was first applied to asteroids by Veverka (e.g., 06.098.024; 09.098.062) and has been extensively utilized by Zellner and collaborators $(12.098 .036 ; 12.098 .086$; and papers in press). The two methods are in reasonable agreement, although the radiometric albedos tend to be systematically lower than the polarimetric, especially for the darkest objects. By and large, asteroids are darker and larger than believed before and span a remarkably wide range in albedos, from 0.02 to 0.4 , with a bimodal distribution tending toward dark $(0.03-0.06)$ and light $(0.10-0.20)$ groups.

\section{Spectral Reflectivities}

Compositionally diagnostic information on asteroids has been derived primarily from photometry with numerous narrow-band filters (usually 24 ) from 0.3 to $1.1 \mu \mathrm{m}$ that has yielded for the first time a large sample of reflection spectra with detailed slopes and recognizable absorption features (Chapman et al., 09.098.004). Comparisons with spectral signatures of minerals and rocks suggest probable identifications; in particular the weak absorption bands can be attributed to pyroxene, olivine and other silicates. Valuable insights into the relationships between asteroids and meteorites have emerged from extensive laboratory measurements of the albedos and spectral reflectivities of meteorites (Egan et al. 10.105.102; Johnson and Fanale, 10.105.106; McCord and Gaffey, Science 186, 352). A catalog of meteorite spectra from 0.3 to $2.5 \mu \mathrm{m}$ has been prepared by Gaffey (JGR, in press). Chapman et al. (Icarus 25, 104), using all polarimetric, radiometric and spectral parameters available for 110 asteroids, have discussed a synthesis of these data. About $90 \%$ of the measured asteroids fall into two broad groups: Group C (very dark), is interpreted as carbonaceous chondrites; Group S (bright reddish) is interpreted as probably stony-metallic meteorites. Rarer types identified include $\mathrm{M}$ objects apparently corresponding to nickel-iron meteorites or possibly metal-rich enstatite chondrites, and $E$ asteroids probably identifiable with the aubrites (iron-depleted enstatite achondrites). Mars-orbit crossing asteroids, including 1685 Toro (Chapman et al., 10.098.002), 433 Eros (Pieters et al., Icarus, in press), and 1566 Icarus (Egan et al., 10.105.002), may be similar to ordinary chondrites. Infrared photometry of a dozen asteroids in the 1 to $3 \mu \mathrm{m}$ range is reported by Johnson et al. and Chapman and Morrison (Icarus, in press). High-resolution Fourier transform IR spectra of Vesta and Eros (Larson et al., Icarus, in press) confirm for Vesta the rapidly cooled basaltic composition suggested by McCord et al. (03.098.019) from visible and near-infrared spectrophotometry. Caldwell (Icarus 25, 384) extends broadband photometry for Ceres, Pallas and Vesta farther into the UV with the OAO-2; the violet decrease in albedo continues down to $2600 \AA$.

\section{Photometric and Polarimetric Surveys}

The broad compositional and albedo classes discussed in (III) and (IV) have been defined primarily in terms of spectrophotometry and albedos derived from radiometry and polarimetry. Recent work has demonstrated, however, that most asteroids can be classified on the basis only of their $U B V$ colors and the minimum value of their linear polarization, $P_{\min }$. Surveys carried out by Zellner and Gradie $(A J$, in press) and Zellner et al. $(A J 80,986)$ extend $U B V$ observations and determinations of $P_{\min }$ to 91 asteroids.

\section{Unusual Individual Asteroids}

Unusual individual asteroids have been revealed by recent observations. 4 Vesta is the only asteroid (down to a survey limit of about $50 \mathrm{~km}$ diameter) with a basaltic surface and hence presumably a differentiated interior, in spite of its being about 5 times less massive than the apparently undifferentiated 1 Ceres. 16 Psyche and 22 Kalliope show featureless, red-sloping spectra and intermediate albedos that suggest they may be primarily metallic in composition. 44 Nysa was found by Zellner $(A p J 198, L 45)$ to have an albedo of 0.4 , the highest of any 
known asteroid; the albedo, together with the spectrum, suggests a composition similar to the aubrites. 349 Dembowska has been identified as the only large main-belt asteroid with a composition like that of an ordinary LL-type chondrite. 324 Bamberga was the first dark (albedo $\sim 0.03$ ) asteroid to be identified, but more recent work shows it to be a fairly typical member of the $\mathrm{C}$ class of asteroids, the largest of which (excluding 1 Ceres and 2 Pallas, both of which have somewhat anomalous spectra) are 10 Hygiea, 704 Interamnia, 65 Cybele, 52 Europa, and 511 Davida. A particularly unusual group is the Trojans; all of those studied have very low albedo, red-sloping spectra in the visible, and unique $U B V$ colors. These physical data indicate the Trojans are distinct from both other asteroids and the outer satellites of Jupiter, and in addition their surfaces do not correspond to any known meteorite types.

\section{Distribution in Belt}

C-type asteroids predominate in the outer portions of the belt (Chapman et al., Icarus, $25,104)$. High-albedo asteroids lacking pyroxene absorptions may be concentrated in the center of the belt along with the darkest objects, resembling $\mathrm{C} 2$ chondrites. Those with pyroxene bands are in the inner part, and the one or two with spectra resembling ordinary chondrites are innermost of all. Chapman (12.098.075) interprets S-type objects to be cores of differentiated precursors, and notes that carbonaceous chondrites seem to be at the largest distances and ordinary chondrites at smaller distances, in agreement with the solar nebula condensate models; however, the interpretation fails for the M-type objects, if they are of enstatite-chondritic composition. Since the asteroid belt is overwhelmingly C-type, it rather supports the large temperature gradient of Lewis (12.107.014). The present heterogeneity in the belt makes Chapman question whether large mixing took place before reaching present orbital stability, or whether most objects were made in situ.

\section{The Size Distribution}

The size distribution was obtained previously by assuming an average albedo. The new diameters deduced from the individual albedos yield different size distributions for $\mathrm{C}$ and $\mathrm{S}$ objects. The $\mathrm{C}$ distribution follows an almost straight line of slope -3 on an incremental $\log -\log$ frequency diagram, suggesting a heavily fragmented population, whereas the $S$ objects show an apparent excess near $150 \mathrm{~km}$ diameter, suggesting a population incompletely fragmented (Chapman, 12.098.075). Since iron is 20 times stronger than stone, itself two orders of magnitude stronger than carbonaceous chondrites, Chapman is tempted to view the $\mathrm{S}$ bodies as iron or stony-iron 'cores' of geochemically differentiated asteroids.

\section{Collisional Evolution}

The larger collisional cross-sections implied by the low albedos require that asteroid collisions were and are much more frequent than previously believed. Indeed, Chapman and Davis (Science 190,553 ) present evidence for an early population, perhaps 300 times as large as the present one, dominantly in C objects of $500 \mathrm{~km}$ or less, that have plausibly cratered the Moon, Mars and Mercury. Vesta is probably the only example of a proto-S object that retains its mantle and crust, since it has a basaltic, hence differentiated surface. Large collisional rates are also implied by Napier and Dodd (11.098.002), Wetherill (11.098.003), and Dorschner (11.098.016). A collision of a small planet with a large comet has a probability much too low to be considered (Horedt 11.098.025). Hirayama families (defined by concentrations of proper orbital elements) show disparate spectral types (McCord and Chapman, ApJ 195, 553) consistent with fragmentation of differentiated primordial bodies. Levin (10.098.072) also discusses the fragmentation of asteroids.

\section{Rotation}

The observed rotation of the main object in Hirayama families is explained by that collision with a smaller body that caused the splitting into the given family (Gerasimov, 12.098.013). McAdoo and Burns (09.098.011) interpret known rotational properties of 64 asteroids from a collisional model. Photoelectric light curves yielding amplitudes and spin rates have been determined by numerous observers (Wamsteker, Sather, Schober, Lustig, Schroll, Scaltriti, 
Zappala, Taylor, Cristescu, van Houten-Groeneveld, Tedesco, etc.). The pole of rotation for 433 Eros has been determined with good precision by Dunlap and by Millis et al. (both Icarus, in press). Taylor $(10.098 .071)$ shows that the period of Vesta is $10^{\mathrm{h}} 41^{\mathrm{m}}$ rather than half this size, as previously thought. Burns and Safronov (10.098.018) calculate that internal energy dissipation aligns the rotation and body axes faster than off-axis collisions misalign them.

\section{$\mathrm{XI}$. Eros and Toro Campaigns}

The Apollo asteroid 1685 Toro passed within $0.14 \mathrm{AU}$ of Earth in the summer of 1972 and was studied by a variety of techniques. Dunlap et al. (10.098.001) determined $U B V$ colors and from measurements of polarization found an albedo of 0.14 and derived an effective mean radius of $2.2 \pm 0.4 \mathrm{~km}$. Spectrophotometry $(10.098 .002,10.098 .003)$ suggested a surface composition similar to the equilibriated L-type chondrites, and Goldstein et al. detected this asteroid by $12-\mathrm{cm}$ radar $(10.098 .004)$. A more extensive campaign was organized by Zellner and Gehrels to study the Mars-crossing asteroid 433 Eros during its close approach ( $0.15 \mathrm{AU})$ in 1973-74, resulting in 15 papers in Icarus (May, 1976) and a popular review by Zellner in Sky and Telescope (Dec., 1975). Radiometry and polarimetry, when combined with photometric light curves, agree on an albedo of 0.19 and mean diameters of a tri-axial ellipsoid of $13 \times 15 \times 36 \mathrm{~km}$. Also determined were the period, sense of rotation, and pole of rotation. Spectrophotometry from 0.3 to $2.1 \mu \mathrm{m}$ reveals a unique spectral reflectance, with a reddish slope probably due to free iron as well as absorption bands near 0.9 and $1.95 \mu \mathrm{m}$ characteristic of pyroxene. Measurements of $10-\mu \mathrm{m}$ brightness as a function of phase show that Eros has a low (lunar-type) thermal conductivity, a result that together with the low radar reflectivity suggests that free iron, if present at the surface, is finely dispersed among silicate grains. The radar returns also indicate that the asteroid is extremely rough at $12-\mathrm{cm}$ wavelength, although thermal and polarimetric evidence requires the presence of a dusty regolith at least a centimeter deep. The closest compositional match seems to be with the iron-rich (H-type) ordinary chondrites.

\section{METEORITES}

\section{A. Introduction}

To save space, only review articles and books are included in the reference list. Papers are cited in the text by author and year and can be traced through the sources given or through the various abstracting services.

Most meteoriticists are still heavily committed to work on lunar samples, although the time period 1973 to 1975 saw steadily increasing activity in meteorite research, largely a result of several exciting discoveries having fundamental significance for astronomers and meteoriticists alike.

Several books appeared in this period, on meteorite research in general (Wasson, 1974) carbonaceous chondrites (Nagy, 1975), iron meteorites (Buchwald, 1975) opaque minerals in stony meteorites (Ramdohr, 1973) and nuclear cosmochemistry (Lavrukhina, 1972).

A well-referenced review of meteorite research carried out between July 1970 and June 1974 was published by Wasson (1975). Other reviews were written on the mineralogy (Mason, 1972) and magnetism (Herndon and Rowe, 1974) of meteorites, magnetic studies done in the Soviet Union (Herndon et al., 1972), $\mathrm{Cr}$ (Bunch and Olsen, 1974a) and organic compounds (Anders et al., 1973a) in meteorites, sources and orbits of meteorites (Wetherill, 1974), condensation processes in the solar nebula (Larimer, 1973a; Grossman and Larimer, 1974; Grossman, 1975a), iron meteorites (Scott and Wasson, 1975) and meteoritic material on the moon (Anders et al., 1973b; Morgan et al., 1975). Fuchs et al. (1973) published a detailed monograph on the Murchison Type 2 carbonaceous chondrite and a collection of papers on the newly-fallen ureilite Haverö appeared in Meteoritics 7, 1972. Several articles were written on the ancient (Bjorkman, 1973) and recent (Fales, 1972; Sears, 1975; Lange, 1975) history of the development of man's knowledge about meteorites. Three issues of Meteoritika (No. 32, 33, 34) were published in the USSR in 1973-75. 


\section{B. Abundances of the Elements}

Estimates of the abundances of non-volatile elements in the solar system continue to be based on analytical data from type 1 carbonaceous chondrites (Cameron, 1973a). Krähenbühl et al. (1973) measured concentrations of 17 trace elements in 8 different samples of these meteorites, showing data that exhibit far less scatter than earlier studies. New data appeared for rare earths (Nakamura, 1974), siderophiles and chalcophiles (Case et al., 1973) and $\mathrm{Li}, \mathrm{Na}$ and $\mathrm{K}$ (Nichiporuk and Moore, 1974) in Orgueil and Ivuna. Ozerova et al. (1973) reported very high concentrations of $\mathrm{Hg}$ in Orgueil compared to expectations for type 1 carbonaceous chondrites. This study, and earlier ones with similar results, may have been plagued by contamination effects related to be ubiquity of $\mathrm{Hg}$ in laboratories, its volatility and the high specific surface area of these meteorites. Analytical difficulties have also hampered measurements of the $\mathrm{Zr}$ and Hf abundances. Ehmann and Chyi (1974) reported new data for these elements, 45\% lower and a factor of 3 lower, respectively, than earlier data. Ganapathy et al. (1976) confirmed this new Hf abundance but found that $\mathrm{Zr}$ is $40 \%$ lower than measured even in that study. Cameron et al. (1973) pointed out the high abundance of $B$ suggested by existing, internally-consistent meteoritic data but Audouze et al. (1973) found that the interstellar $B$ abundance is at least a factor of 5 lower.

\section{Chondrites and Condensation}

Using the new solar system abundance table (Cameron, 1973a) and estimates of the nebular $P$ and T conditions (Cameron and Pine, 1973), Grossman $(1975 \mathrm{~b}, \mathrm{c})$ updated earlier calculations of the condensation sequence at high temperatures. Lewis (1974) showed that the densities of the terrestrial planets and condensation temperatures of their components are consistent with the nebular temperature gradient derived by Cameron and Pine (1973). Kelly and Larimer (1976) computed, as a function of temperature, the concentrations of 15 siderophile elements in metallic nickel-iron as it equilibrates its composition with a solar gas. Larimer (1975) showed that graphite, $\mathrm{CaS}$, carbides and nitrides would replace $\mathrm{Ca}-$, Al-rich silicates and oxides as the highest temperature condensates if the nebular $\mathrm{C} / \mathrm{O}$ ratio exceeded 0.9 and suggested that the enstatite chondrites may have formed from such a system. Arrhenius and De (1973) warned against the assumption of thermal equilibrium between grains and gas in condensation models.

Considerable attention was focused on mineralogical and chemical characteristics of the coarse-grained, high-temperature $(>1450 \mathrm{~K})$ condensate inclusions in the Allende type 3 carbonaceous chondrite. A large number of refractory trace elements, in spite of radically different chemical properties and geochemical behavior, were all found to be enriched in these inclusions (Grossman, 1973; Tanaka and Masuda, 1973; Martin and Mason, 1974; Mason and Martin, 1974; Wänke et al., 1974; Chou et al., 1976). The data of Grossman and Ganapathy (1976a) revealed considerable detail about the condensation behavior of these refractories. Mineralogical, petrographic and chemical studies concluded that some of the inclusions were once molten (Grossman and Clark, 1973; Fuchs, 1974; Mason and Martin, 1974; Blander and Fuchs, 1975; Grossman, 1975d; Kurat et al., 1975; Grossman and Ganapathy, 1976a). Another type of inclusion in Allende, the fine-grained pink variety, is thought to have formed when refractory elements remaining in the gas after condensation of the coarse-grained inclusions condensed at low temperature $(<1200 \mathrm{~K})$ together with volatiles (Grossman and Ganapathy, $1975 ; 1976 \mathrm{~b})$. The textures of these inclusions were studied by Grossman et al. (1975) and their chemistry by Tanaka and Masuda (1973), Martin and Mason (1974), Boynton (1975) and Grossman and Ganapathy $(1975,1976 \mathrm{~b})$. Grossman and Stele (1976) described inclusions of yet another type from Allende, the amoeboid olivine aggregates, which appear to be mechanical mixtures of high-temperature condensates with low-temperature olivine.

Olsen et al. (1973), Grossman and Olsen (1974) and Olsen and Grossman (1974) showed that textural and chemical properties of much of the olivine and metal in the type 2 carbonaceous chondrite Murchison are consistent with their having condensed at about $1400 \mathrm{~K}$. It appears that types 2 and 3 carbonaceous chondrites contain a remarkable array of different petrographic components which represent the products of a complex history of condensation, 
melting, agglomeration and accretion processes during cooling of the solar nebula.

Masuda et al. (1973), Nakamura and Masuda (1973) and Nakamura (1974) reported irregularly-fractionated rare earth element patterns in carbonaceous and ordinary chondrites and attributed them, at least in part, to chemical fractionations during the condensation of chondritic matter. Highly irregular rare earth patterns are common in components of the Allende meteorite (Tanaka and Masuda, 1973; Grossman and Ganapathy, 1976a, b).

Larimer (1973b) improved earlier estimates of condensation temperatures of the highly volatile elements $\mathrm{Pb}, \mathrm{Bi}$, In and $\mathrm{Tl}$ and,together with Laul et al. (1973), computed accretion temperatures on the order of $420-540 \mathrm{~K}$ for individual ordinary and enstatite chondrites from their depletions in these elements. Dodd (1974) cautioned against this approach, claiming that the grain size and abundance of the metallic phase into which $\mathrm{Pb}, \mathrm{Bi}$ and $\mathrm{Tl}$ condensed may be significantly different from those assumed by the former workers, leading to higher and discordant inferred condensation temperatures. Blander (1975) has also criticized volatile element cosmothermometry, disagreeing with Anders and Larimer (1975) on the magnitude of uncertainties in the thermodynamic data and on the justification of assuming equilibrium at these low temperatures. The use of the oxygen isotope cosmothermometer, which previously had given the same accretion temperatures as volatile trace elements, has become complicated (Onuma et al., 1974) because of the discovery of chondritic oxygen isotope anomalies produced by nuclear effects.

Wasson and Chou (1974) claimed to have found evidence for nebular dissipation during the condensation of moderately volatile elements but Anders (1975a) disputed this conclusion because of the poor quality of the elemental abundance data upon which it was based and conflicting information from calculated condensation temperatures. Fanale and Cannon (1974) proposed that planetary rare gases were incorporated in Orgueil by adsorption from the nebular gas.

Controversy over the origin of chondrules persists. Suggestions favoring a primary mode of origin include equilibrium condensation of liquid droplets in the high P-T environment of the pre-collapse stage of the proto-planetary atmosphere of Jupiter (Podolak and Cameron, 1974) and non-equilibrium condensation of metastable, subcooled liquid droplets (Blander and Fuchs, 1975 ) at the lower pressures of the solar nebula. Cameron (1973b) proposed that chondrules formed in a secondary process, by crystallization of melt droplets generated during impacts between dust grains with velocities greater than $1 \mathrm{~km} \mathrm{~s}^{-1}$. Lange and Larimer (1973) claimed petrographic evidence for such a process but this was questioned by Vedder and Gault (1974). Kieffer (1975) favored the impact hypothesis, suggesting that pre-chondrule dust particles had diameters of $0.5 \mathrm{~mm}$ to $20 \mathrm{~cm}$.

\section{Ages}

Wetherill et al. (1973) reported a very low initial $\mathrm{Sr}^{87} / \mathrm{Sr}^{86}$ ratio from a high-temperature condensate inclusion in Allende with a low $\mathrm{Rb} / \mathrm{Sr}$ ratio. Gray et al. (1973) found another such inclusion with the lowest $\mathrm{Sr}^{87} / \mathrm{Sr}^{86}$ ever seen in meteorites, indicating that it, and presumably others like it, could have condensed from the solar nebula before the bulk of chondritic matter, in agreement with earlier mineralogical and chemical studies. The U-Pb systematics of these inclusions also suggest that they are extremely ancient (Tatsumoto et al., 1976) but in this study and in that of Gray et al. (1973) evidence was found for relatively recent disturbance of the isotopic systems.

Tilton (1973) reported $\mathrm{Pb}$ isotopic compositions for several chondrites and suggested that isotopic lead ages for Bruderheim and Pultusk may be concordant. Huey and Kohman (1973) and Tatsumoto et al. (1973) used $\mathrm{Pb}$ isotopic measurements to show that individual chondrites have distinctly different model ages, within a range of about $50 \times 10^{6} \mathrm{yr}$ and that some achondrites may be slightly older than some chondrites. Cameron (1973c) suggested that these apparent age differences may be artifacts due to incomplete isotopic mixing of the nebular gas, leading to different initial $\mathrm{Pb}$ isotopic compositions for different bodies.

$\mathrm{Sm}^{147}$ decays to $\mathrm{Nd}^{143}$ with a half-life of $1.06 \times 10^{11} \mathrm{yr}$. Lugmair and Scheinin (1975) used this pair to define an internal isochron for the heavily shocked Stannern eucrite, obtaining an 
age of $4.46 \pm 0.12 \times 10^{9} \mathrm{yr}$ where other dating schemes give a variety of younger ages. This age agrees with the internal $\mathrm{Rb}$-Sr isochron for the Juvinas eucrite (Allègre et al., 1975) which shows that it last underwent igneous crystallization $4.60 \pm 0.07 \times 10^{9} \mathrm{yr}$ ago.

Application of the $\mathrm{Rb}-\mathrm{Sr}$ and $\mathrm{Ar}^{40}-\mathrm{Ar}^{39}$ techniques to Ca-rich achondrites shows that they have multi-stage histories and often record relatively young events. Nakhlites underwent an igneous or metamorphic re-heating episode 1.2 to $1.4 \times 10^{9}$ yr ago (Podosek, 1973; Papanastassiou and Wasserburg, 1974; Gale et al., 1975). Podosek and Huneke (1973) showed that some eucrites lost radiogenic $\mathrm{Ar}^{40}$ in the time period 3.5 to $4.4 \times 10^{9} \mathrm{yr}$ ago. Howardites appear to be regolith breccias, containing igneous clasts with different crystallization ages (Papanastassiou et al., 1974) and glass fragments which lost $\mathrm{Ar}^{40}$ after igneous crystallization, presumably as a result of meteorite impacts on parent bodies (Kirsten and Horn, 1975; Rajan et al., 1975).

\section{E. Extinct Radionuclides}

From measured I-Xe ages, Herzog et al. (1973) and Lewis and Anders (1975) showed that magnetite from the Orgueil and Murchison carbonaceous chondrites is the oldest meteoritic material dated so far. Karoonda, a metamorphosed carbonaceous chondrite, began to retain radiogenic $\mathrm{Xe}^{129} 1.8 \pm 0.5 \times 10^{6} \mathrm{yr}$ later, implying that its parent body accreted within $10^{6} \mathrm{yr}$ of magnetite condensation.

Chemical fractionation of $\mathrm{Pu}$ from $\mathrm{U}$ continues to hamper the use of fissiogenic Xe from extinct $\mathrm{Pu}^{244}$ for dating purposes. The initial $\mathrm{Pu}^{244} / \mathrm{U}^{238}$ ratio inferred for the early solar system, 0.015 , is based on Xe measurements of the St. Severin chondrite. Lewis (1975) expressed concern over the use of this value after noting that the $\mathrm{Pu}^{244} / \mathrm{U}^{238}$ ratio was higher and variable in whitlockite separated from this chondrite. He pointed out that the fission $\mathrm{Xe}$ is correlated more strongly with rare earths than with U. Crozaz (1974) found that Th was enriched more than $U$ in the whitlockite compared to bulk St. Severin, suggesting that $\mathrm{Pu}$ followed Th rather than U. Podosek and Lewis (1972) observed an I-Xe age similar to St. Severin for early condensate inclusions from Allende but their high initial $\mathrm{Pu}^{244} / \mathrm{U}^{238}$ ratio, 0.087 , suggests much earlier formation than St. Severin. Shirck (1974) failed, however, to detect the extraordinary concentration of fission tracks that would be expected from this much $\mathrm{Pu}$.

For a given mineral, Pellas and Storzer (1975) found that the density of $\mathrm{Pu}^{244}$ fission tracks decreases with increasing metamorphic grade in ordinary chondrites. They suggested that metamorphism was simultaneous with accretion and that the more highly metamorphosed chondrites accreted at higher temperatures and took longer to cool below track annealing temperatures than the unequilibrated ones.

A novel interpretation of the radiogenic Xe anomalies, with potentially far-reaching consequences for early solar system chronology, was proposed by D. D. Clayton (1975). He suggested that the decay of extinct nuclides occurred largely before the condensation of the solar system, inside interstellar grains which were later incorporated into chondrites.

Notsu et al. (1973) claimed to have found large excesses of $\mathrm{Nd}^{142}$ in the Juvinas achondrite and proposed that they were due to decay of extinct $\mathrm{Sm}^{146}$ with a half-life of $1.03 \times 10^{8} \mathrm{yr}$. When Lugmair et al. (1975) performed the same experiment with much higher precision, however, $\mathrm{Nd}^{\mathbf{1 4 2}}$ anomalies were not detected.

Gray and Compston (1974) and Lee et al. (1976) detected $\mathrm{Mg}^{26}$ excesses in the high-Al, low-Mg, high-temperature condensate inclusions in Allende and attributed them to in situ decay of extinct $\mathrm{Al}^{26}$ which has a half-life of $0.72 \times 10^{6} \mathrm{yr}$. At present, however, there are too few data points to tell whether the anomalies are due to $\mathrm{Al}^{26}$ or to incorporation of interstellar grains with non-solar $\mathrm{Mg}$ isotopic compositions (see next section). If the $\mathrm{Al}^{26}$ hypothesis is confirmed by future measurements, these observations could be of fundamental significance in establishing that this long-sought-after radioactive heat source had not totally decayed before the condensation of the chondrites.

Lewis et al. (1975) and Anders et al. (1975) achieved a major breakthrough toward indentifying excess $\mathrm{Xe}^{134}$ and $\mathrm{Xe}^{136}$ in carbonaceous chondrites as fission products of an extinct, superheavy element. They successfully isolated an acid-resistant residue, amounting to $<1 \%$ of the 
Allende meteorite, which contains $50 \%$ of the anomalous Xe. This fraction is also enriched in the volatiles $\mathrm{Tl}, \mathrm{Bi}$ and $\mathrm{Pb}$ which are predicted to have similar nebular condensation temperatures as their congeners in the periodic table, elements 113 to 115 . Previously seen only in temperature release fractions, the anomalous $X e$ is also enriched in non-fission $X^{124}$ and $\mathrm{Xe}^{126}$, a fact which had caused Manuel et al. (1972) to doubt the fission hypothesis. In Lewis $e t$ al. (1975), however, there is the hint of separation between light and heavy Xe isotopes. Kuroda et al. $(1974,1975)$ suggested that the Xe anomalies are not fissiogenic but were produced in a complex series of processes, including a mass-dependent fractionation.

\section{F. Isotope Anomalies and Interstellar Grains}

Perhaps the most exciting development in cosmochemistry in the period 1973-1975 was the discovery by Clayton et al. (1973) that oxygen in anhydrous minerals of types 2 and 3 carbonaceous chondrites consists of two components having different isotopic compositions. The exotic component, enriched in $\mathrm{O}^{16}$ compared to terrestrial oxygen, is thought to have entered the solar nebula in the form of refractory interstellar grains which were incompletely evaporated during its high-temperature stage and incorporated by chondritic minerals as they condensed from the vapor. Subsequent study (Clayton et al., 1975) revealed that this component is heterogeneously distributed inside the condensate inclusions, indicating lack of homogenization since the anomalies were established. That study, together with Clayton and Mayeda (1975), showed that the Earth, Moon, achondrites and enstatite chondrites all contain the same amount of the exotic component and could have formed from the same reservoir within the solar nebula. The $\mathrm{H}$-group ordinary chondrites contain less of the exotic component than the earth and the L-group even less, indicating a different formation location for each and for carbonaceous chondrites.

The discovery of oxygen isotope anomalies triggered the search for anomalies in isotopic compositions of other light elements. Lee and Papanastassiou (1974) found small, but measurable, deviations from the terrestrial $\mathrm{Mg}^{24} / \mathrm{Mg}^{26}$ ratio in several inclusions from the Allende meteorite. These variations do not correlate with the $\mathrm{Al} / \mathrm{Mg}$ ratios of the inclusions, indicating that the decay of nowextinct $\mathrm{Al}^{26}$ to $\mathrm{Mg}^{26}$ is not the cause of these magnesium anomalies. Nor do they correlate with the oxygen isotopic variations, suggesting that more than one interstellar component is present. Eberhardt (1974) succeeded in isolating a small fraction of the silicates in the type 1 carbonaceous chondrite Orgueil which contains large enrichments in neon-E, a component rich in $\mathrm{Ne}^{22}$. Eberhardt suggested that the host of this component, previously seen enhanced only in temperature-release fractions from Orgueil (Herzog and Anders, 1974), is interstellar in origin. This is not an unreasonable suggestion since Orgueil may have formed in a part of the nebula whose peak temperature did not exceed $400^{\circ} \mathrm{K}$. Neon may have been unable to diffuse out of interstellar grains in such low-temperature regions which are predicted in Cameron and Pine's (1973) model nebula.

\section{G. Metamorphosed and Differentiated Meteorites}

Chondrite investigations continue into progressive changes with increasing metamorphic grade of textures (Fredriksson et al., 1975), Major (Bunch and Olsen, 1974b; Larimer and Buseck, 1974) and trace (Chou and Cohen, 1973; Chou et al., 1973) element contents of minerals and volatile trace element contents of bulk meteorites (Ikramuddin and Lipschutz, 1975; Ikramuddin et al., 1975).

Studies of achondrites dealt with radioactive element contents (Fisher, 1973; Morgan and Lovering, 1973), petrography (Wilkening, 1973; Brownlee and Rajan, 1973, Noonan, 1974; Lovering, 1975; Wilkening and Anders, 1975) and solar wind and solar flare irradiation history (Rajan and Price, 1973; Macdougall et al., 1973; 1974; Poupeau et al., 1974; Rajan, 1974). From their many similarities with lunar soils, gas-rich meteorites (Wilkening and Clayton, 1974) appear to have been derived from regoliths of their parent bodies. Anders (1975b), noting that there are many solar gas-rich carbonaceous chondrites, suggested that they would have had to remain so long in the asteroid belt to develop a regolith that a cometary origin for them is improbable. 
Studies of stony-irons focused on the chemistry and mineralogy (Mason and Jarosewich, 1973; Wasson et al., 1974) and shock history (Jain and Lipschutz, 1974) of mesosiderites and deformation of pallasitic olivine (Klosterman and Buseck, 1973). Iron meteorite studies concentrated on structure (Goldstein and Axon, 1973; Scott, 1973; Scott and Bild, 1974), chemistry (Scott et al., 1973; Rambaldi et al., 1974), C isotopic composition (Deines and Wickman, 1973; 1975), strength (Auten, 1973; Remo and Johnson, 1974; Johnson and Remo, $1974 ; 1975$ ) and formation pressures (Schwarcz et al., 1975).

\section{H. Miscellaneous}

Concentrations of $\mathrm{N}$ (Kothari and Goel, 1974), Sn (De Laeter et al., 1974), Cd and Zn (Rosman and De Laeter, 1974) and Be (Quandt and Herr, 1974) were determined in bulk meteorites, suites of elements were studied in unequilibrated ordinary chondrites (Kurimoto et $a l ., 1974$; Baedecker and Wasson, 1975), carbonaceous chondrites (Van Schmus and Hayes, 1974) and ureilites (Bogard et al., 1973a; Binz et al., 1975b) as well as in individual chondrules (Osborn et al., 1973; 1974) and separated phases (Allen and Mason, 1973).

The recent fall of Murchison provided a large amount of relatively uncontaminated carbonaceous chondrite material for studies of indigenous organic compounds (Folsome et al., 1973; Lawless, 1973; Levy et al., 1973; Yuen and Kvenvolden, 1973; Hayatsu et al., 1975; Pereira et al., 1975; Pollock et al., 1975). They seem to have formed below $400^{\circ} \mathrm{K}$ in the solar nebula by Fischer-Tropsch reactions using condensate grains as catalysts. Anders $e t$ al. (1974) suggested that this process, acting in dense circumstellar envelopes, is responsible for production of organic molecules now found in interstellar gas.

Research in cosmic ray interactions with meteoritic material focused on variation in production rates of spallogenic nuclides with target element chemistry (Bogard and Cressy, 1973; Nyquist et al., 1973; Trivedi and Goel, 1973; Herzog, 1975; Imamura et al., 1975; Smith and Huneke, 1975) and with depth and size (Wright et al., 1973; Heimann et al., 1974; Herzog and Cressy, 1974; Cressy, 1975). New data on cosmogenic nuclides in individual meteorites were reported by Bogard et al. (1973b), Ganapathy and Anders (1973), Smith and Fireman (1973), Wilkening et al. (1973), Bibron et al. (1974), Cameron and Top (1974), Cressy and Rancitelli (1974), and Levsky and Komarov (1975).

Studies of magnetism in meteorites (Brecher and Arrhenius, 1974; Rowe, 1975) have been unable to determine time of magnetization or whether the fields were generated inside or outside of the parent bodies.

\section{REVIEWS AND BOOKS ON METEORITES}

Anders, E., Hayatsu, R., and Studier, M. H.: 1973a, Science 182, 781.

Anders, E., Ganapathy, R., Krähenbühl, U., and Morgan, J. W.: 1973b, The Moon 8, 3.

Bjorkman, J. K.: 1973, Meteoritics 8, 91.

Buchwald, V. F.: 1975, Handbook of Iron Meteorites, Univ. Calif. Press, Berkeley (in press).

Bunch, T. E. and Olsen, E.: 1975, Geochim. Cosmochim. Acta 39, 911.

Fales, H. B.: 1972, Meteoritics 7, 237.

Fuchs, L. H., Olsen, E., and Jensen, K. J.: 1973, Smithson. Contrib. Earth Sci. 10.

Grossman, L.: 1975a, Sci. Am. 232, 30.

Grossman, L. and Larimer, J. W.: 1974, Rev. Geophys. Space Phys. 12, 71.

Herndon, J. M. and Rowe, M. W.: 1974, Meteoritics 9, 289.

Herndon, J. M., Rowe, M. W., Larson, E. E., and Watson, D. E.: 1972, Meteoritics 7, 263.

Lange, E. F.: 1975, Meteoritics 10, 241.

Larimer, J. W.: 1973a, Space Sci. Rev. 15, 103.

Lavrukhina, A. K.: 1972, Nuclear Reactions in Cosmic Bodies (in Russian), Nauka Publ. Co., Moscow.

Mason, B.: 1972, Meteoritics 7, 309.

Morgan, J. W., Ganapathy, R., Higuchi, H., and Anders, E.: 1975, in Proc. Soviet-Amer. Conf. on Cosmo-

chem of Moon and Planets (in press).

Nagy, B.: 1975, Carbonaceous Meteorites, Elsevier Scientific Publ. Co., Amsterdam.

Ramdohr, P.: 1973, The Opaque Minerals in Stony Meteorites, Elsevier Scientific Publ. Co., Amsterdam. 
Scott, E. R. D. and Wasson, J. T.: 1975, Revs. Geophys. Space Phys. 13, 527.

Sears, D. W.: 1975, Meteoritics 10, 215.

Shukolukov, Yu. A. and Leyskij, L. K.: Geochemistry and Cosmochemistry of Rare Gas Isotopes (Moscow, 1972).

Wasson, J. T.: 1974, Meteorites-Classification and Properties, Springer-Verlag, New York.

Wasson, J. T.: 1975, Rev. Geophys. Space Phys. 13, 113.

Wetherill, G. W.: 1974, Ann. Rev. Earth Planet. Sci. 2, 303.

\section{CONCISE REFERENCE SYSTEM}

Most papers quoted in $A A A$ are referenced by their 2 digit $A A A$ volume number, followed by their $A A A$ reference number. However, many papers related to the same comet are hidden in $A A A$ under the same reference number. To facilitate retrieval, the original reference has then been preferred. References not from the $A A A$ are by volume and page, (no year, no author's initials, no more than 2 authors' names; abbreviations used are given hereafter).

\begin{tabular}{|c|c|c|c|c|}
\hline$A A$ & - & Astron. Astrophys & $B A I C$ & - Bull. Astron. Inst. Czech. \\
\hline$A A A$ & & Astron. Astrophys. Abstracts & $B L g$ & Bull. Soc. Royale Sci. Liège \\
\hline & & Astronomia i Astrofizika, Kiev & $C J P$ & Can. J. Phys. \\
\hline$A R A A$ & & Ann. Rev. Astron. Astrophys. & $C R$ & Compt. Rend. Acad. Sci. Paris \\
\hline$A J$ & - & Astron. J. & $D A T$ & Dokl. Akad. Nauk Tadjik U.S.S.R. \\
\hline ApJ & - & Astrophys. J. & $K M D$ & Komety i Meteory, Dushanbe \\
\hline$A L$ & - & Astrophys. Letters & $K T K$ & Kometny Tsirk., Kiev \\
\hline$A S S$ & & Astrophys. Space Sci. & $M N$ & Monthly Notices Roy. Astron. Soc. \\
\hline$A T$ & - & Astronomich. Tsirk., Moskva & $P A S P$ & - Publ. Astron. Soc. Pacific \\
\hline$A V$ & & Astronomich. Vestn. & $P K F$ & - Problemi Kosmicheskoj Fiziki, Kiev \\
\hline$A Z$ & - & Astron. Zh. (Akad. Nauk S.S.S.R.) & PSS & Planetary Space Sci. \\
\hline$B A A S$ & & Bull. Am. Astron. Soc. & $Q J R A S$ & - Quart \\
\hline Cl & & \multirow{5}{*}{\multicolumn{3}{|c|}{$\begin{array}{l}\text { NASA's Workshop, Oct. 10-11, 1973, Albuquerque, N.M., U.S.A. } \\
\text { NASA's Workshop, June 13-14, 1974, Huntsville, Ala., U.S.A. (NASA SP-355, 1975) } \\
\text { Sixth Lunar Conference } \\
\text { Eighth Conference on Cometary Problems, Kiev } 1974 \\
\text { IAU Colloquium No. 12, 'Physical Studies of Minor Planets,' March 6-10, 1971, Tucson, Ariz., } \\
\text { U.S.A. (NASA SP-267, 1971). }\end{array}$}} \\
\hline$C 2$ & - & & & \\
\hline C6 & & & & \\
\hline C8 & & & & \\
\hline C12 & & & & \\
\hline C2O & & \multirow{3}{*}{\multicolumn{3}{|c|}{$\begin{array}{l}\text { Twentieth International Astrophysical Colloquium, Liège, June 17-20, } 1975 . \\
\text { IAU Colloquium No. 22, 'Asteroids, Comets, Meteoritic Matter,' April 4-6, 1974, Nice, France } \\
\text { IAU Colloquium No. } 25 \text { 'The Study of Comets,' Oct 28-Nov. 1, 1975, Greenbelt, Md., U.S.A., } \\
\text { NASA SP-393. }\end{array}$}} \\
\hline$C 22$ & & & & \\
\hline C25 & & & & \\
\hline C31 & & \multicolumn{3}{|c|}{$\begin{array}{l}\text { IAU Colloquium No. } 31 \text { 'Interplanetary Dust and Zodiacal Light,' June 10-13, 1975, Heidel- } \\
\text { berg, F.R.G. }\end{array}$} \\
\hline C39 & - & \multirow{2}{*}{\multicolumn{3}{|c|}{$\begin{array}{l}\text { IAU Colloquium No. 39, 'Relationships between Comets, Minor Planets and Meteorites,' } \\
\text { Aug. 17-20, 1976, Lyon, France }\end{array}$}} \\
\hline & & & & renoble, France, $\mathrm{Au}$ \\
\hline
\end{tabular}

A. H. DELSEMME President of the Commission 